\title{
1 The influence of objecthood on the representation of natural images in
}

\section{the visual cortex}

4 Paolo Papale ${ }^{1,2, \neq}$, Wietske Zuiderbaan ${ }^{3, \neq}$, Rob. R.M. Teeuwen ${ }^{1}$, Amparo Gilhuis ${ }^{1}$, Matthew W. Self ${ }^{1,9}$,

5 Pieter R. Roelfsema ${ }^{1,4,5, \uparrow,{ }^{*}}$ and Serge O. Dumoulin ${ }^{1,3,6,7,9}$

6

1. Department of Vision \& Cognition, Netherlands Institute for Neuroscience (KNAW), 1105 BA Amsterdam, Netherlands

2. Momilab Research Unit, IMT School for Advanced Studies Lucca, 55100 Lucca, Italy

3. Spinoza Centre for Neuroimaging, 1105 BK Amsterdam, Netherlands

4. Department of Integrative Neurophysiology, VU University, 1081 HV Amsterdam, Netherlands

5. Department of Psychiatry, Academic Medical Centre, Postbus 22660, 1100 DD Amsterdam, Netherlands.

6. Department of Experimental and Applied Psychology, VU University Amsterdam, Amsterdam 1181 BT, Netherlands

7. Department of Experimental Psychology, Helmholtz Institute, Utrecht University, 3584 CS

$19 \neq$ shared first author contribution

I shared senior author contribution

\footnotetext{
* correspondence should be addressed to: p.roelfsema@nin.knaw.nl
} 


\section{Abstract}

Neurons in early visual cortex are not only sensitive to the image elements in their receptive field but also to the context determining whether the elements are part of an object or background. We here assessed the effect of objecthood in natural images on neuronal activity in early visual cortex, with $\mathrm{fMRI}$ in humans and electrophysiology in monkeys. We report that boundaries and interiors of objects elicit more activity than the background. Boundary effects occur remarkably early, implying that visual cortical neurons are tuned to features characterizing object boundaries in natural images. When a new image is presented the influence of the object interiors on neuronal activity occurs during a late phase of neuronal response and earlier when eye movements shift the image representation, implying that object representations are remapped across eye-movements. Our results reveal how object perception shapes the representation of natural images in early visual cortex.

\section{Introduction}

The visual scenes that we perceive are filled with objects. We readily identify the extent of the objects and their boundaries, a perceptual organization process that is important for our understanding of an image's meaning. Accordingly, the judgments of people who are asked to mark regions occupied by objects and their boundaries are highly consistent ${ }^{1}$. Object and boundary perception even influence low-level vision, because image elements at object boundaries are better perceived than image elements at less relevant image locations ${ }^{2,3}$. Furthermore, image elements of objects have a higher perceived contrast than those that are part of the background ${ }^{4}$. Despite these influences on low-level visual perception, it is not yet well understood how objecthood influences neuronal representations in early visual cortex ${ }^{5}$. 
Classical descriptions of the activity of neurons at the early levels of the visual system focus on the features that drive neurons, such as the contrast and orientation in a neuron's receptive field (RF). In addition, there are also non-classical, contextual influences on neuronal activity, which originate from outside the neurons' RFs and play a role in the grouping of features into objects. Here we focus on two such effects: boundary modulation (BoM) related to the detection of object boundaries, and object-background modulation (OBM) related to the grouping of object features into objects and their segregation from the background.

Neurons in the primary visual cortex (V1) and area V4 increase their firing rate when their RF is centered on an elongated contour that extends well beyond their RF (Fig. 1a) $)^{6,7}$. Elongated contours are relevant for perceptual organization because they usually signal the borders of objects in natural scenes, whereas shorter contours are more likely to be part of the background ${ }^{8,9}$. BoM is the extra activity elicited by contours that demarcate object boundaries (Fig. 1a,d). Similarly, V1 and V4 neurons exhibit stronger responses when their RF falls on the interior of a perceptual object than when it falls on the background (Fig. 1b) ${ }^{10-12}$. This OBM occurs for all image regions that are part of an object, suggesting that the response enhancement could cause the binding of the distributed representation of features in early visual cortex into coherent perceptual objects ${ }^{13}$. This view is supported by the finding that objects relevant for behavior elicit stronger OBM than objects that are not, implying a relation between $\mathrm{OBM}$ and object-based attention that co-selects all features of a relevant object ${ }^{11}$. BoM and $\mathrm{OBM}$ are thought to reflect the recurrent interactions within and across visual areas ${ }^{14}$ that determine the spread of enhanced neuronal activity and thereby the perception of spatially extended objects in a scene ${ }^{13}$.

So far, BoM and OBM have only been measured with artificial stimuli, such as textures and displays with many line elements (Fig. 1a-c). Establishing the relevance of these signals for natural vision is 
68

69

70

71

72

73

74

75 are remapped across eye movements in early visual cortex ${ }^{17,18}$.

challenging, yet important, because neuronal response properties that do not play a role in the perception of natural stimuli are likely to be of limited relevance ${ }^{15}$. A recent study explored contextual signals in V1 elicited by more complex shapes, such as the texture-defined ' $U$ ' of Figure $1 c^{16}$, but researchers have, to our knowledge, not yet examined BoM and OBM with natural visual stimuli. If BoM occurs in natural images, we predict that the more salient object boundaries elicit stronger neuronal activity than image elements of the background. Similarly, if OBM occurs for natural images, extra activity should be elicited by object interiors compared to the background.

To investigate the influence of objecthood on neuronal activity in early visual cortex, we used the Berkeley Segmentation data set (BSD), a library of natural images in which human observers marked object boundaries ${ }^{1}$. We used functional MRI to examine neuronal responses across many regions of visual cortex in humans and we also recorded multi-unit activity in V1 and V4 of monkeys to gain insight into the temporal profile of spiking activity. We report that objecthood influences neuronal activity. Object boundaries increased the early neuronal responses and object interiors enhanced activity during a later phase of the response. When subjects made eye movements across the images, these contextual effects carried over from one fixation to the next, implying that objects 
a

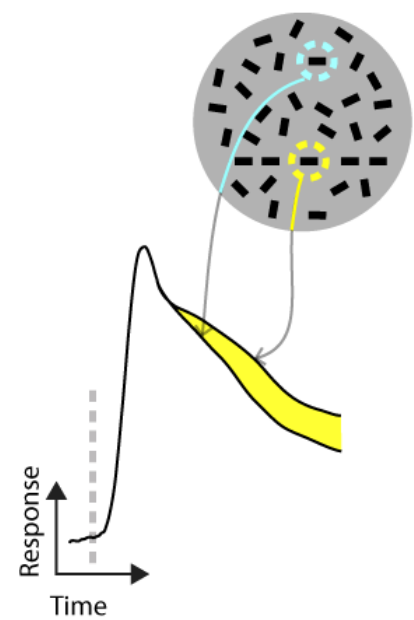

e

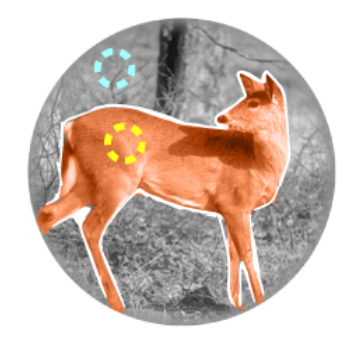

b

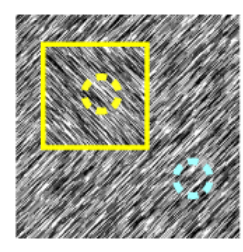

C

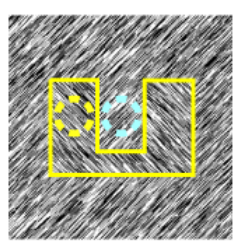

d

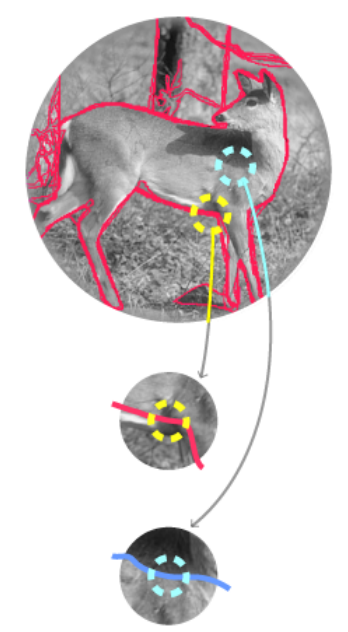

f

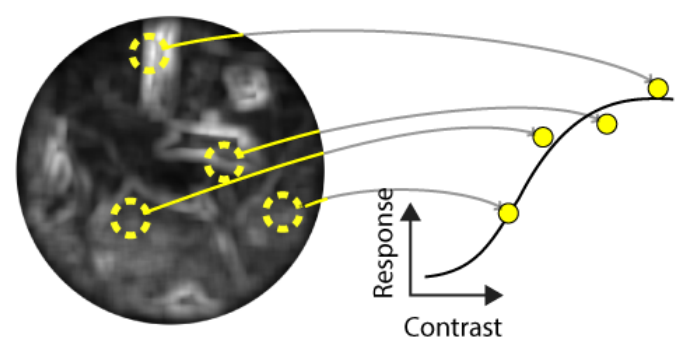

OBM (Fig. 1e) from the influence of the contrast of image elements by evaluating the influence of

Figure 1. The influence of object perception on neuronal responses in early visual cortex.

$\mathrm{a}$, The response of $\mathrm{V} 1$ and $\mathrm{V} 4$ neurons is enhanced when their RF falls on an elongated contour that extends well beyond their $\mathrm{RF}^{6} . \mathbf{b}, \mathbf{c}, \mathrm{V} 1$ and $\mathrm{V} 4$ neurons exhibit stronger responses when their $\mathrm{RF}$ falls on the interior of a perceptual object (square with different orientation in $b$ and ' $u$ ' shape in $c$ ) than when it falls on the background ${ }^{16}$. $\mathbf{d}, \mathbf{e}$, We ask whether differences between object borders (yellow in d) and other image regions (cyan in d) and between the interiors of objects (yellow in e) and the background (cyan in e) in natural images influence the response of visual cortical neurons. $\mathbf{f}$, We compared the response amplitudes evoked by image elements of objects and the background, taking the local contrast in the ( $p)$ RF into account.

\section{Results}

Objecthood modulates responses in human early visual cortex

In the first experiment, we used ultra-high field fMRI at 7 Tesla in four human participants to investigate OBM and BoM within natural images (Fig. 2). Our analysis separated BoM (Fig. 1d) and image properties in the population receptive field (pRF) on the neuronal responses at each cortical 
location. We chose 45 images from the BSD (Fig. S1) for which the object boundaries had been annotated by an independent group of human observers ${ }^{1}$.

To separate the influence of image contrast from object perception we computed the contrast response functions (CRFs; Fig. 1f) for six regions of interest: V1, V2, V3, human V4 (hV4), the lateral occipital visual field maps $1 / 2$ (LO-1/2) and V3-a/b. First, in a separate experiment, we measured the population receptive field for each cortical location (pRF; Fig. S2) ${ }^{19}$. Second, to compute the CRF, we estimated the response amplitude as function the root mean square (RMS) contrast in each $\operatorname{pRF}^{20}$ (10 contrast bins, Fig. 1f).

Next, we computed the CRF separately when the pRF fell on an object border versus a non-border image region, and when it fell an object region versus on the background for every image (Fig. $2 b)^{1,21}$. We defined object borders in the BSD images as those that were frequently marked by the observers as object boundaries, and contrasted them to non-border image regions that were not marked (Fig S3). These non-border regions could be part of the object interior (as the example in Fig. 1d and Fig. S3) or background (see Methods).

The computation of the CRF allowed us to separate BoM and OBM from the influence of contrast. Cortical responses elicited by object borders were significantly higher than those elicited by nonborder image regions in areas V1, V2 and V3 (Fig. 2c; all ps $<0.001$, bootstrap test, see Methods), but not in areas V3ab, hV4 and LO-1 and 2 (Fig. S2). Thus, we observed significant BoM in V1-V3. Object boundaries of a particular contrast elicit a larger response, on average, than image regions with the same contrast that do not coincide with object boundaries.

To examine the influence of OBM, we compared CRFs of cortical locations with pRFs on object versus background regions (Fig S3). The response amplitude when a pRF was centered on an object was 
significantly stronger than when it was centered on the background in V1 and V2 (Fig. 2d; all ps < results also at the level of individual participants, for both BoM and OBM (Figure S4).

We conclude that object borders elicit larger response amplitudes in early visual cortex than non-

a
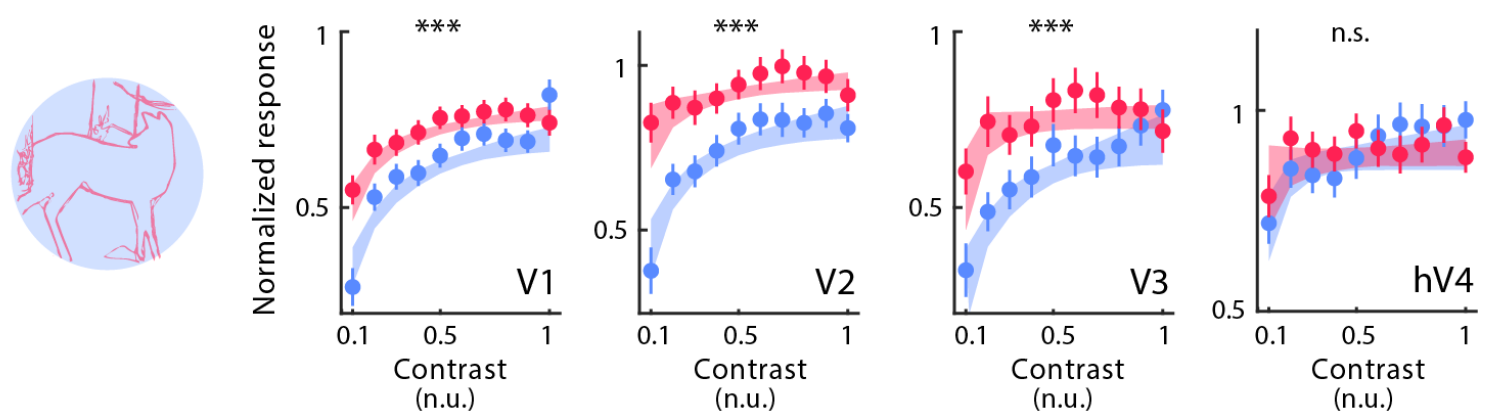

b
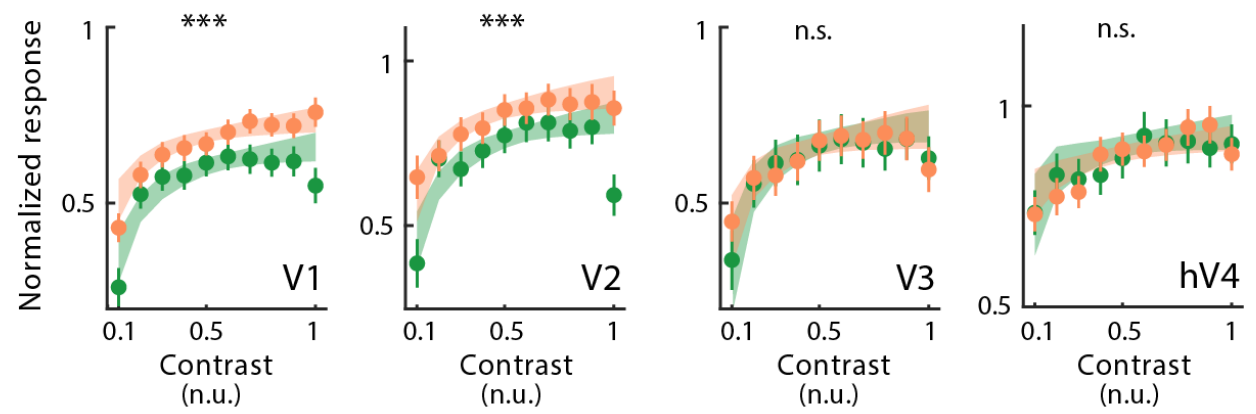

Figure 2. Objecthood influences responses in human early visual cortex.

Four participants viewed 45 natural images while their brain responses were recorded with fMRI. a, Response amplitudes elicited by object boundaries (red) and background contours (blue) as a function of contrast in the pRF ( $x$ axis). $\mathbf{b}$, Response amplitudes elicited by object interiors (orange) and the background (green). $\mathrm{FMRI}$ responses were normalized to the response to a full-field, $100 \%$ contrast stimulus. Shaded regions denote $95 \%$ confidence intervals determined by bootstrapping. Bars represent SEM across images ( ${ }^{* * *}$ indicates $p<0.001$, bootstrap test; $n$.s. non-significant). 
Objects and their boundaries enhance the spiking activity of V1 and V4 neurons but at different latencies

A limitation of $\mathrm{fMRI}$ is its poor temporal resolution and its indirect relation to spiking activity 22 .

143 Therefore, we recorded spiking activity with chronically implanted electrode arrays elicited by BSD

144 stimuli in two macaque monkeys. We placed the arrays in areas V1 and V4 and recorded multi-unit 145 spiking activity (MUA). Whereas the pRFs in the MRI experiment covered the entire images, the RFs 146 of the V1 and V4 neurons in the electrophysiology experiment were confined to a limited region of 147 the visual field. To increase the sample of image patches falling in the RFs, we trained the monkeys to fixate at multiple locations on a total of four images (Fig. 3a).

149 At the start of the trial, the monkey directed gaze to a fixation point on a gray background. We presented the image once the monkey had maintained fixation for $300 \mathrm{~ms}$. After a delay of $400 \mathrm{~ms}$, we presented a new fixation point and the monkey made a saccade to it and maintained fixation for 152 a further $400 \mathrm{~ms}$ (Fig. 3a). The repositioning of gaze was repeated for a total of 6 positions (sampled 153 from a uniformly spaced grid with 500 points) per trial. The neuronal response elicited by the image appearing at the first gaze position differs from that for later fixations because the image suddenly appears in the RF. Later fixations are preceded by saccades causing a rapid movement of part of the image through the RF. Furthermore, the image is now familiar and the monkeys may have recognized and segmented the objects during previous fixations. We therefore separately analyzed

158 the response elicited by stimulus onset (Fixation 1) and later fixations (Fixations 2-6). The results for 159 fixations 2-6 were comparable and we therefore pooled the data across these fixations (Fig. S5). 
a
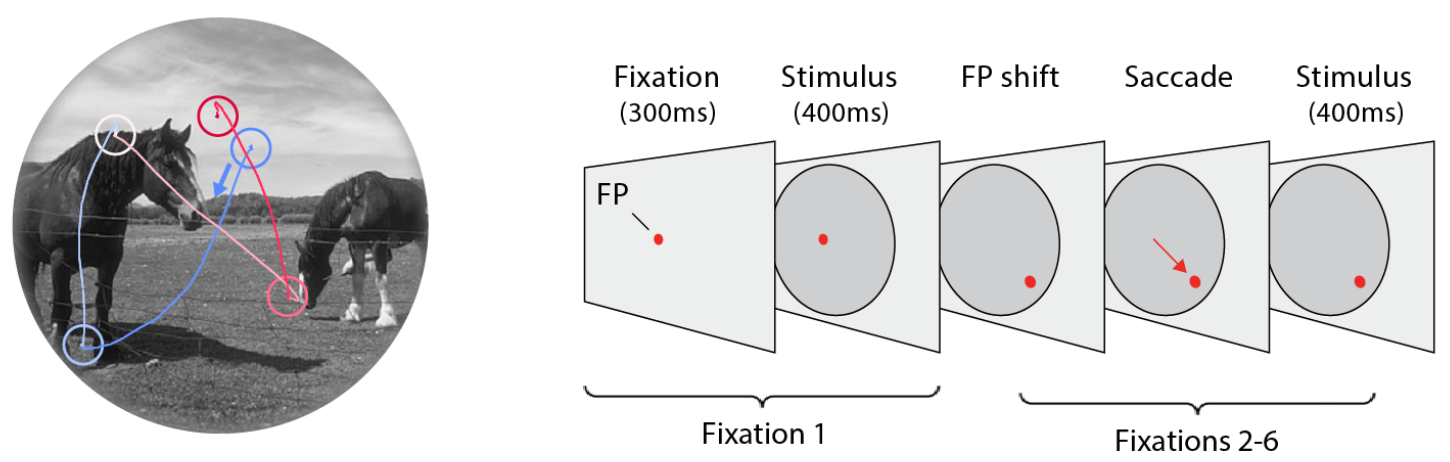

b

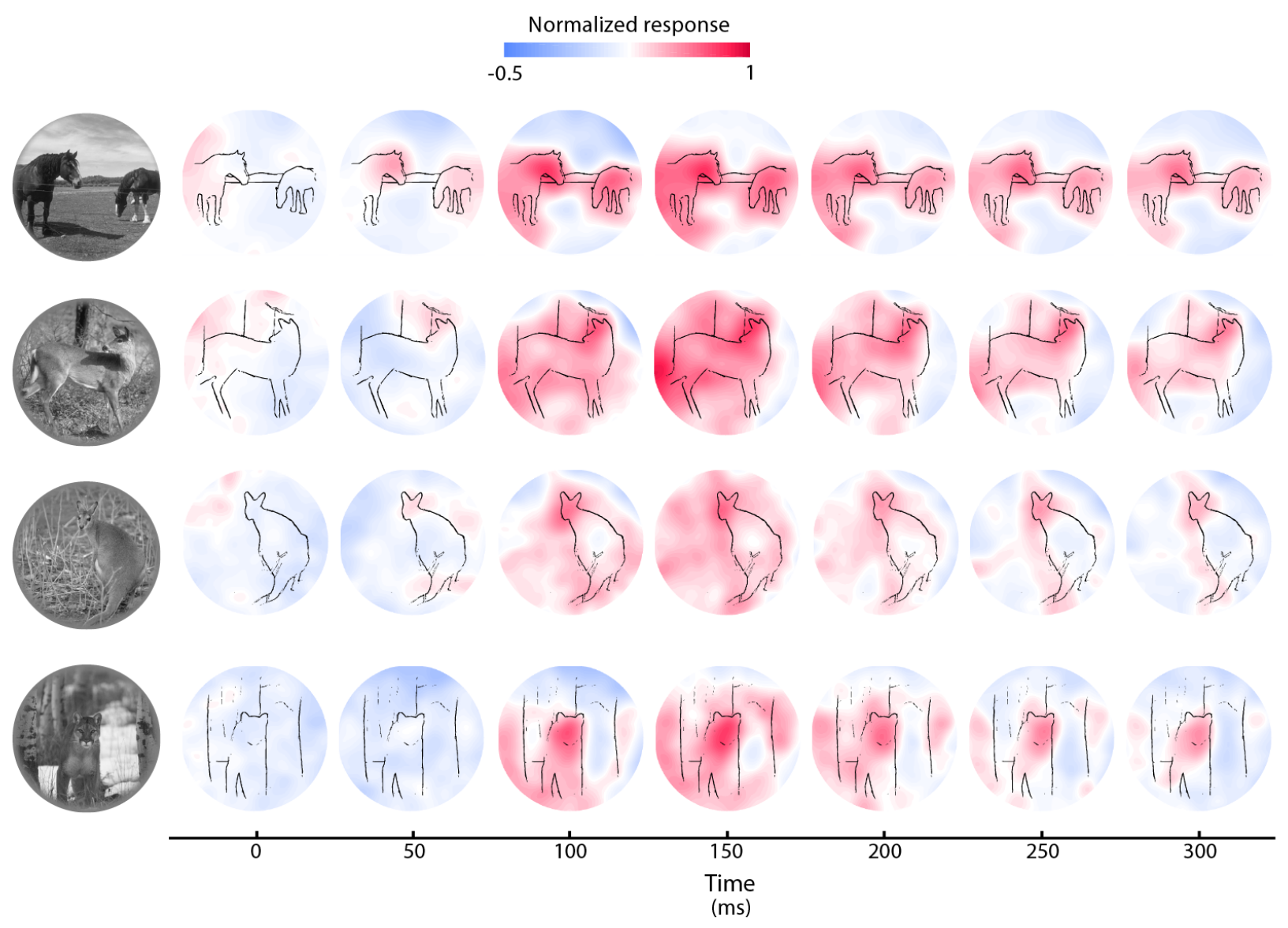

Figure 3. Influence of objects on spiking activity in areas V1 and V4 evoked by natural scenes.

a, The monkey performed a sequence of eye-movements across the natural images. We presented a natural image once the monkey had maintained gaze on a red fixation point for $300 \mathrm{~ms}$. After a delay of $400 \mathrm{~ms}$, a new fixation point appeared and the monkey made a saccade to it and maintained fixation for a further $400 \mathrm{~ms}$. Per trial, the monkey made a total of 5 eye movements to fixation points sampled from a uniformly spaced grid ( 500 points). b, Overlay of V4 spiking activity over the natural images at different time points (average of fixations 2-6). V4 response is determined by contrast, local and global image structure and these factors were disentangled in subsequent analyses. Fig. S3b shows the same analysis for fixation 1 in V4 and fixation 1 and 2-6 in V1. 
We first examined the overall level of activity in V1 and V4 elicited by the four natural images (Fig. influences of objecthood and contrast were not yet separated in this analysis. To disentangle the influence of BoM and OBM from that of contrast, we determined CRFs by binning the contrast in the RF of neurons in area V1 (77 recording sites, 44 in monkey B and 33 in monkey M) and V4 (22

177 sites in monkey B), separately for contours that demarcate object boundaries and those that do not.

178 Object borders elicited stronger spiking activity (time-window 0-300ms) than non-object image 179 regions with the same contrast in V1 and V4 (Fig. 4a). BoM occurred during the first fixation as well as during later fixations (all ps $<0.001$, bootstrap test) and was present at many V1 recording sites in monkey B (fixation 1, 66\% of the sites; fixation 2-6, 77\%; Fig. S6) and monkey M (fixation 1, 45\%; borders and non-border image regions, averaged across contrast bins. We estimated latency as the time-point at which the fitted function reached the $33 \%$ of its maximum (see Methods) $11,12,23$. In V1, the BoM latency was $50 \mathrm{~ms}$ in both fixation 1 and in later fixations. BoM latency was not significantly different from the latency of the visually driven response (49ms for fixation 1 and 29ms for later fixations), neither for fixation 1 nor for the later fixations (both $p s>0.05$, bootstrap test). The same was true for V4 in both conditions (BoM: $59 \mathrm{~ms}$ for fixation 1; 49ms for fixations 2-6; onset of response: $61 \mathrm{~ms}$ for fixation $1 ; 54 \mathrm{~ms}$ for fixations $2-6$; both ps $>0.05$, bootstrap test). We cannot directly compare latencies between fixation 1 and later fixations, because in fixation 1 the image replaced a grey background whereas the image moved through the receptive fields preceding the later fixations. We corrected for this difference by computing $L_{a} t_{B o M-V i s}$, the difference between the 
195

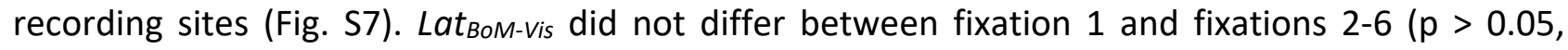
Wilcoxon signed-rank test; Fig. S7).

Next, we compared the response elicited by object interiors to that elicited by background regions (Fig. 4b). Regions that were part of objects elicited more activity in V1 and V4 than background regions, both during the first fixation and later fixations (all ps $<0.001$, bootstrap test). Many V1 recording sites exhibited OBM (monkey B, fixation 1, 41\%, fixations 2-6, 52\%; monkey M, fixation 1, $67 \%$, fixations $2-6,36 \%$ of sites with $p<0.05$, bootstrap test) and OBM was also present in V4 (monkey B, fixation 1, 41\% of sites in V4, fixations $2-6,45 \%$ ). Hence, OBM also occurs for natural images: image elements of objects elicit a stronger activity than those that are part of the background.

In V1, the latency of OBM during the first fixation was $78 \mathrm{~ms}$, which was later than the onset of the visually driven response ( $p<0.05$, bootstrap test). We next examined whether the OBM latency was shorter for later fixations, because the monkeys may have segmented the image in figure and background during the previous fixations. Interestingly, the median OBM latency across sites for fixations 2-6 was only $61 \mathrm{~ms}$, and not significantly different from the visually driven response and BoM ( $p>0.05$, bootstrap test). To correct for the difference in visual stimulation we computed

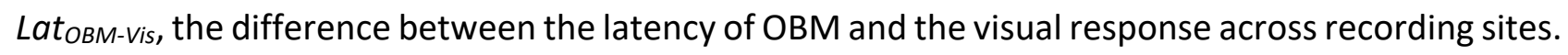
In V1, Lat signed-rank test; Fig. S7). In V4, the latency of OBM was later than the onset of visually driven response both for fixation 1 and fixations 2-6 (both ps $<0.05$, bootstrap test). The difference in Lat this was caused by the smaller number of V4 recording sites. 
217 The earlier OBM in V1 during fixations 2-6 suggests that it may have carried over from earlier

218 fixations during which the monkeys had already recognized and segmented the objects, in 219 accordance with previous studies demonstrating that image segmentation results can be remapped across saccades ${ }^{17,18}$. 
a

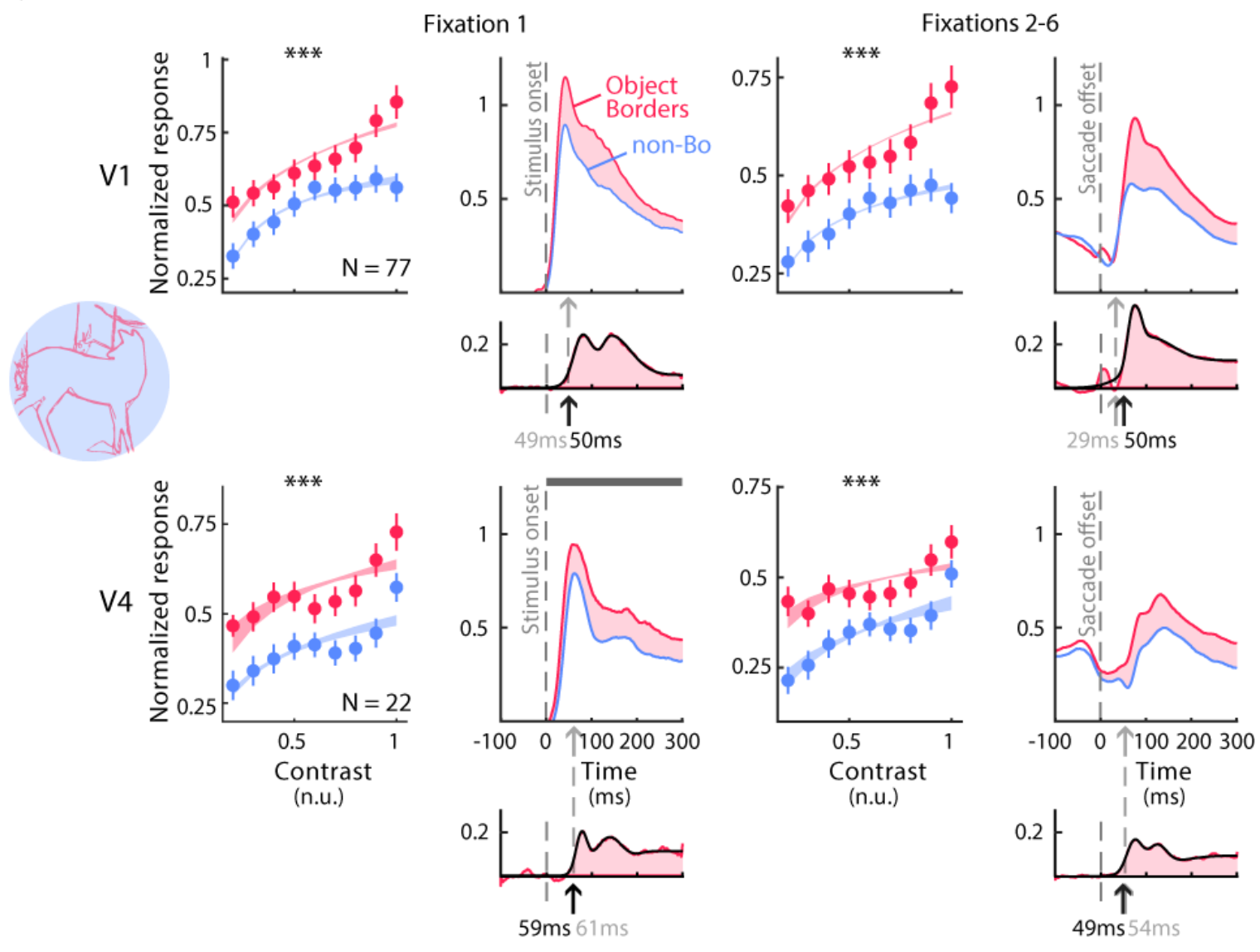

b

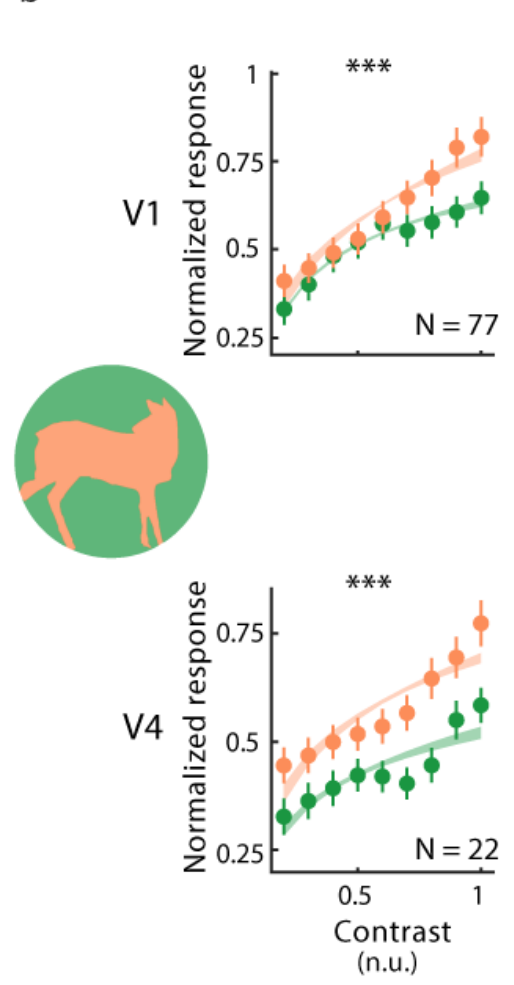

221
Fixation 1
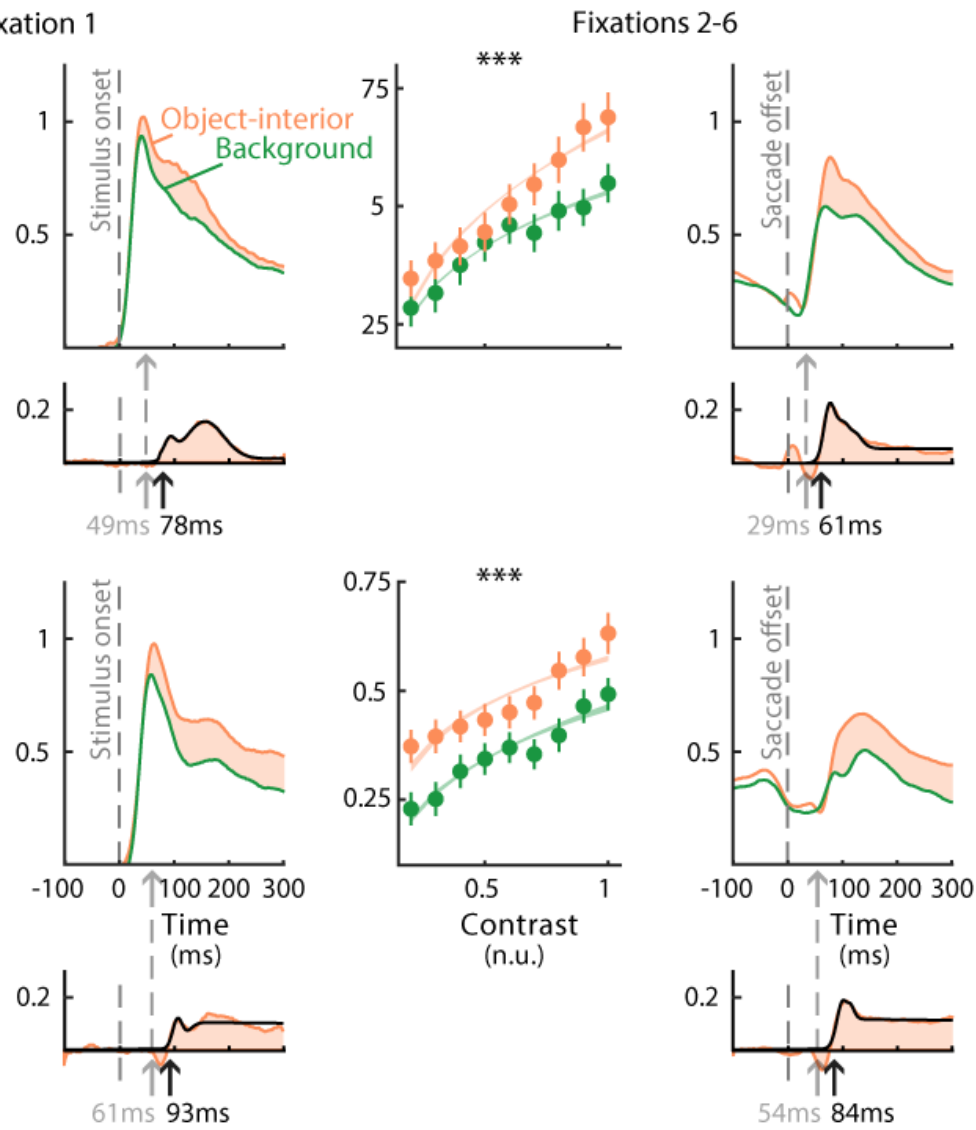
Figure 4. Object borders and their interiors enhance the spiking activity of V1 and V4 neurons relative to background regions.

a, Average CRFs (left) and MUA time-courses (averaged across contrast bins; right) in V1 (top row) and V4 (bottom row) for object-borders (red) and non-border image regions (blue). BoM is significant in both areas. Shaded regions around the CRFs denote 95\% confidence intervals (determined by bootstrapping). Error bars indicate SEM across recording sites $(* * *, p<0.001$, bootstrap test). b, CRFs and MUA time-course elicited by the object-interior (orange) and background (green). Black arrows indicate the latency of either BoM/OBM and gray arrows the latency of the visually driven response.

BoM entails a comparison of the response elicited by object borders and other image elements, which were inside the objects or in the background, whereas OBM entails a comparison of image elements inside objects to background elements (Fig S3). BoM and OBM are not independent because both measures compare neuronal activity evoked by some of the object elements and background elements. We therefore also investigated the amount of unique variance in the activity of V1 and V4 neurons (time window $0-300 \mathrm{~ms}$ ) explained by BoM, OBM and contrast (Fig. 5a; see Methods). Each predictor explained a significant amount of unique variance in both areas and both fixation conditions (all ps $<0.001$, t-test). In V1, contrast explained $41.0 \%$ of the variance, BoM $8.3 \%$ and OBM 5.5\% during the first fixation and the values increased slightly to $44.8 \%, 10.1 \%$ and $6.4 \%$ for fixations 2-6, respectively. In V4, contrast explained $19.0 \%$, BoM $21.4 \%$ and OBM $16.0 \%$ of the variance during the first fixation and these values were $20.8 \%, 21.1 \%$ and $35.0 \%$ for the later fixations. Hence, BoM and OBM accounted for a significant fraction of the explainable variance. Contrast explained less variance in V4 than in V1 whereas the contributions of BoM and OBM were larger in V4. It is of interest that the fraction of variance explained by OBM in V4 increased from $16 \%$ for the first fixation to $35 \%$ for the later fixations. This result suggests that the extra activity elicited by the interior of objects builds up across between the first presentation of the image and subsequent eye movements, possibly because the scene is already known. 
251 We were surprised to find BoM in V1 at a latency of 50ms (Fig. 4a), because it is much earlier than 252 the latency of $\sim 95 \mathrm{~ms}$ typically observed for elongated contours in synthetic images ${ }^{7}$. The longer 253 BoM latency of previous studies is compatible with an effect of feedback from higher cortical areas 254 to V1, but a latency of 50ms might be too short for such a feedback loop. An important difference 255 between the present approach and previous studies with synthetic images is that we did not equate 256 the features of contours that form the boundaries of objects and those that were in the background, 257 even though contrasts were matched. We therefore hypothesized that object contours in natural 258 images have other features, on average, than background contours ${ }^{9,24}$, which could explain the early 259 BoM. In other words, some V1 neurons might be tuned to the features of object contours and extract them in their feedforward response, driven from within the RF.

261 We exploited recent advances in artificial neural networks (ANNs) to study the tuning of V1 262 recording sites and to examine if it can account for the extra activity elicited by object boundaries ${ }^{25-}$ $263{ }^{27}$. As a model for V1 tuning we chose layer conv3_1 of VGG-19 (and several other models, Fig. S8), 264 which is the state of the art in predicting V1 responses to natural images ${ }^{28,29}$, and used a two-stage convolutional mapping to take both the spatial and feature selectivity of neurons at individual recording sites into account (see Methods) ${ }^{26}$. We confirmed previous studies ${ }^{28,29}$ demonstrating that the ANN approach for the modeling of V1 tuning outperforms previous models (Fig. S8). To gain 268 insight into the tuning of the V1 neurons and visualize their preferred features, according to the 269 ANN model, we examined the synthetic images that maximized the model response. Figure $5 c$ 270 illustrates a few of these synthetic images (for illustration purposes; we did not present these 271 images as stimuli). We then applied these RF models to an independent set of 100 natural images 272 that had been annotated by human observers to examine if they indeed predicted extra activity for 
object boundaries (Fig. 5b,c and S9). Specifically, we filtered the unseen images with the RF models

For every recording site we determined their border detection performance (BoP), a measure that

Y-axis in Fig. 5c). BoP is an accuracy score that takes the uneven class distribution of salient and non-

V1 tuning detected objected contours above chance level ( $p s<0.05$, permutation test), which indicates that $\mathrm{V} 1$ tuning is indeed useful for boundary detection.

What is the relation between BoM elicited in V1 by the four pictures of our electrophysiological experiments and the BoP of the same recording sites for a different set of images? We computed

in Fig. 5c), across recording sites. The correlation coefficient was 0.25 ( $p=0.037$, t-test), indicating that V1 neurons that express BoM at an early latency are tuned to low-level feature differences that discriminate between object and non-object contours (Fig. 5c).

We next examined how much information about object contours is present across the recorded population of V1 neurons. We built a binary classifier based on the early (25-75ms) V1 activity in response to trials with object-contours or elements with the same contrast in the neurons' RF. To ensure that complex patterns signaling the presence of an object (e.g., the entire head of an animal) could not be detected by the RFs, we only included the 19 recording sites with smallest RFs $\left(<1.5^{\circ}\right)$ in this analysis. Classification of object boundaries during single fixations had an accuracy of $73.5 \%$, 293 which is well above the chance level of $50 \%$ (Fig. $5 d$, top, red bar; $p<0.001$, bootstrap test). Interestingly, when we used the activity of the entire conv3_1 layer of the VVG-19 ANN to detect object-borders the accuracy was similar (Fig. 5d, bottom, red bar; 66.4\%, $p<0.001$, bootstrap test). 
296 Hence, object-contours can be detected with a reasonable accuracy based on local information in 297 individual RFs.

298 We carried out an extra experiment to confirm that the early BoM reflects V1 tuning rather than a contextual influence. We removed the context by copying circular image patches from the BSD that matched the V1 MUA RFs in size onto a grey background. We chose patches with a similar RMS contrast that did or did not contain an object border and centered them on the RFs of neurons at 50 recording sites in monkey B (Fig. 5e). As predicted, patches with object borders elicited a slightly stronger V1 response than patches without object borders with the same contrast $(p<0.001$, Wilcoxon signed rank test; Fig. 5f,g). Hence, the tuning of V1 neurons indeed explains a fraction of the extra activity elicited by object boundaries.

Our finding that BoM is partially explained by $\mathrm{V} 1$ tuning begs the question of a possible contribution of V1 tuning to OBM, i.e. the extra activity by the object interior. We therefore also examined lowlevel differences between image elements of objects and backgrounds and built a binary (linear) classifier to discriminate between object and background regions, based on the early (25-75ms) response of the same $19 \mathrm{~V} 1$ recording sites as above, using trials with the same contrast. The classification accuracy during single fixations was $69.9 \%$ (Fig. $5 d$, top, orange bar; $p<0.001$, bootstrap test) and it was in the same range for the conv3_1 layer of VVG-19 (Fig. 5d, bottom, orange bar; $74.0 \%, p<0.001$, bootstrap test). Thus, even though OBM emerges later than BoM, the activity of a small number of V1 neurons is enough to differentiate between features that characterize the interior of objects and the background. 
a
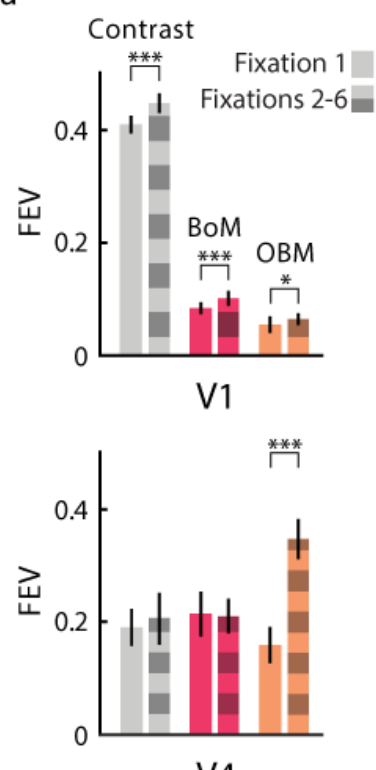

V4 b

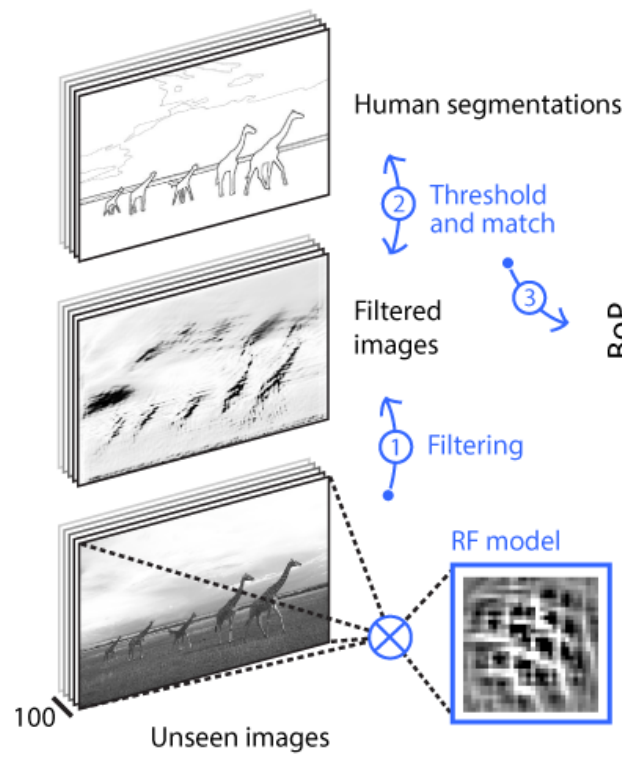

C

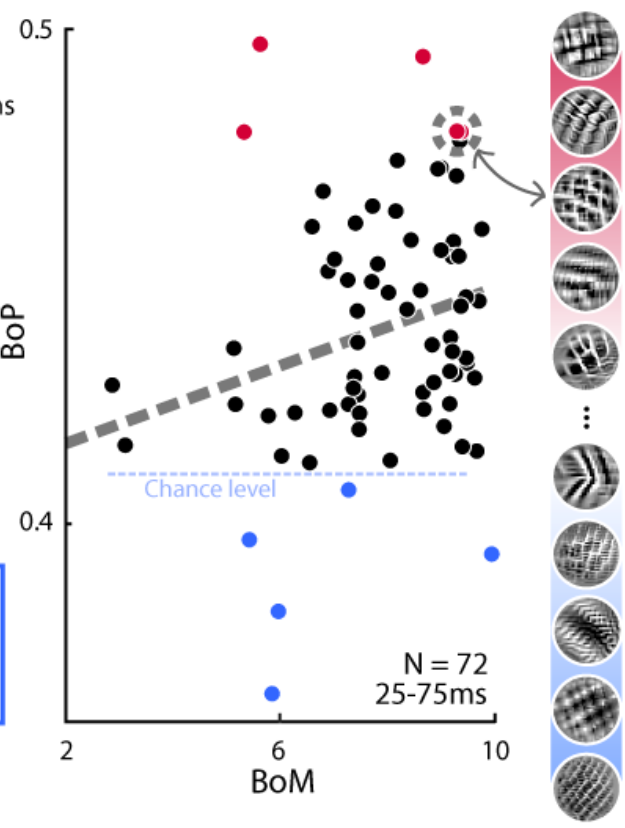

d
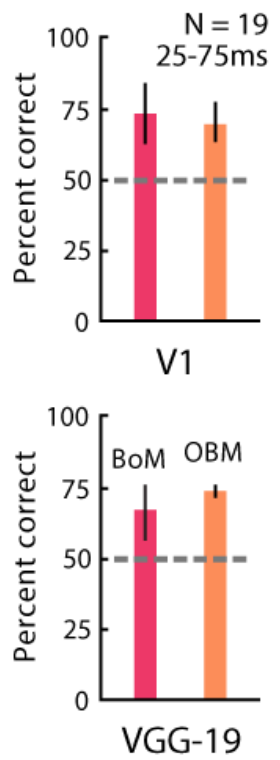

e
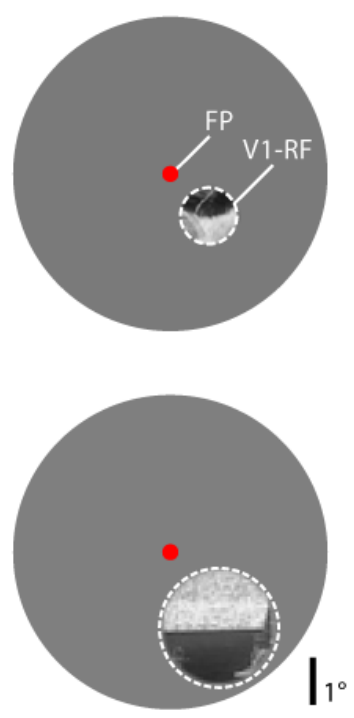

f

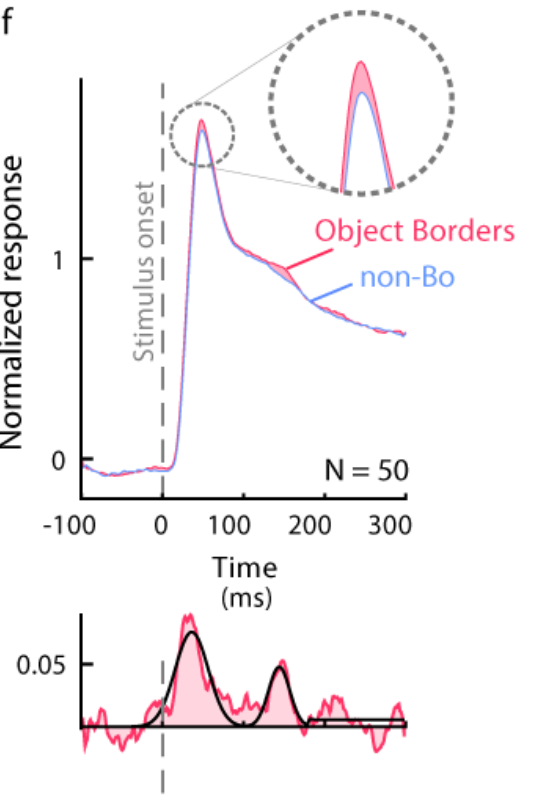

g

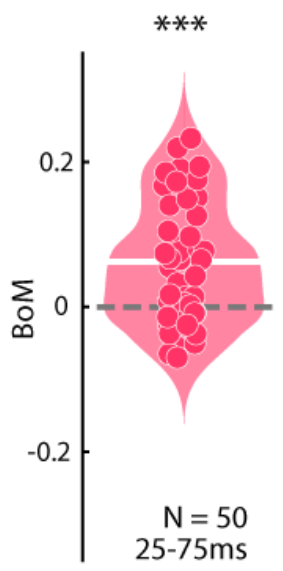

Figure 5. Explained variance in V1 and V4 and V1 tuning during the onset response.

a, Fraction of variance of V1 and V4 activity explained by contrast, BoM and OBM (0-300ms time window) for fixation 1 and fixations 2-6 $\left(^{* * *}: \mathrm{p}<0.0011^{*}: \mathrm{p}<0.05\right.$, Wilcoxon signed rank). Error bars denote SEM. $\mathbf{b}$, We derived RF models for each V1 recording site using ANNs, and applied them to a separate annotated set of natural images to examine how well they can detect object borders. We calculated a measure of border-detection performance (BoP) for every V1 recording site. Step 1 , applying the V1 tuning to the image. Step 2, thresholding of activity and correlation with human judgements. Step 3, measurement of BoP of the V1 recording site. c, Correlation between BoM ( $x$ axis) and BoP ( $y$-axis) across $V 1$ recording sites ( $p<0.05$, parametric test). Blue dashed line, significance threshold for border detection ( $p<0.05$, permutation test). $d$, Accuracy of binary 
classifiers of object contours (red bar) and interiors (orange bar) based on the early response (25$75 \mathrm{~ms}$ ) of 19 V1 sites (upper panel) or the VGG-19 conv3_1 layer (lower panel). Classifiers detected object contours and interiors above chance level. Error bars denote 95\% confidence intervals (determined by bootstrapping). e, Example isolated BSD image patches matching RFs of different $\mathrm{V} 1$ recording sites. $\mathrm{f}$, Time-course of the $\mathrm{V} 1$ responses. Object borders elicited stronger early activity than non-border image patches. $g$, Distribution of early $(25-75 \mathrm{~ms})$ BoM elicited by image patches across recording sites (white bar indicate the median BoM; ${ }^{* *}, \mathrm{p}<0.001$, Wilcoxon signed-rank test).

\section{Contextual BoM in natural images}

The early BoM in natural images is driven by the information in the RF. It differs from BoM in previous studies $^{6,7}$, in which it was a contextual effect driven by information outside the neurons'

RF. Does BoM also occur for natural images if the image elements in the RFs are kept the same? In a further experiment, we placed the RF of $98 \mathrm{~V} 1$ recording sites (68 in monkey B and 30 in monkey M) on object borders and other locations in 12 natural images from the BSD, while keeping the image patch in the RF constant (Fig. 6a). Specifically, we copied an image patch with an object border and pasted it at a background location to create a condition in which the same image patch is not perceived as object border. An example image is shown in Fig. 6a (left panel) where we copied a part of the back of the elephant into the background. On average, the object contours elicited a stronger V1 response than the same image patches presented at background locations $(p<0.001$, Wilcoxon signed-rank test across recording sites; Fig. 6b). The latency of BoM in this experiment was $81 \mathrm{~ms}$, i.e. it now occurred during the delayed phase of the V1 response.

As a control, we placed the image patch at identical locations of synthetic metamers of these images. The metamers had the same orientations, phases, spatial frequencies, auto- and crosscorrelations and marginal statistics, but the layout of objects was scrambled ${ }^{30}$. In the example metamer of Fig. 6a (middle and right panels), the transitions between water, trees and air were at the same locations but the elephant was removed (other example metamers are shown in Fig. S10). 
355 BoM was absent for the metamers ( $p>0.05$, Wilcoxon signed-rank test). To investigate if the level 356 of BoM differed between the metamers and the original images, we performed a repeated357 measures two-way ANOVA with object-border and scrambling (2 levels each) as factors. The main 358 effects of object-borders and scrambling were both significant (salience, $F_{1,97}=28.6, p<0.001$; 359 scrambling, $\left.F_{1,97}=5.42, p=0.022\right)$. Importantly, the interaction was also significant at the population 360 level $\left(F_{1,97}=6.74, p=0.011\right)$ and at many of the individual recording sites $($ at $p<0.05 ; 40 \%$ of the 361 sites in monkey B and $73 \%$ in monkey M). Hence, if RF stimulus is kept constant, contextual 362 information enhances the $\mathrm{V} 1$ activity elicited by object borders, at a latency of $\sim 80 \mathrm{~ms}$. These results, 363 taken together, indicate that there are two processes that jointly explain the enhanced activity 364 elicited by object boundaries. The tuning of V1 neurons enhances their representation from an early 365 time point onwards and the scene context causes an additional activity increase at a longer latency. 
a
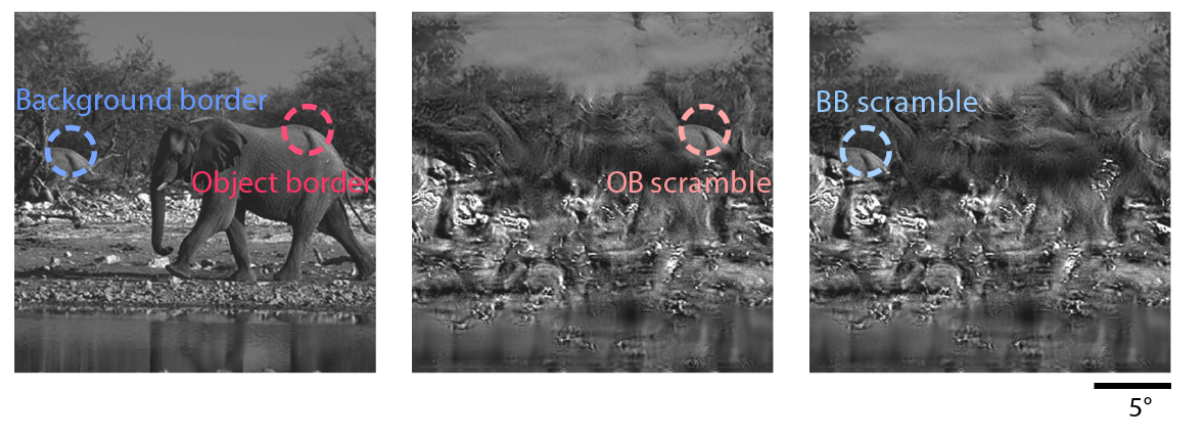

b

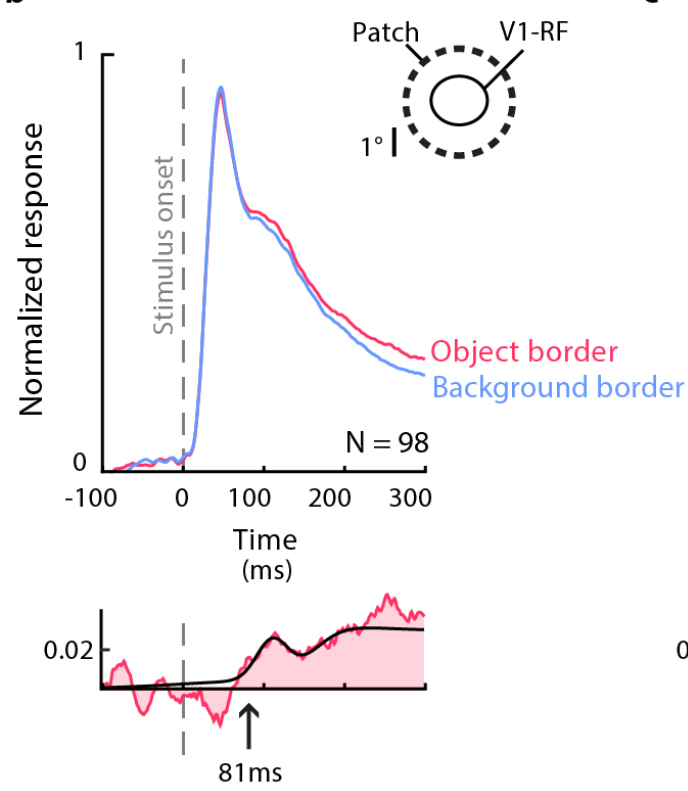

C

Figure 6. Contextual BoM in V1.

a, To examine the role of contextual information around the V1 RF, we modified natural images ensuring that the same features were present the RFs. We either copied an image patch with an object border to a background location (left panel) or removed the object from the scene by creating metamers (middle and right panel). The RF stimulus was kept constant across all the conditions. b, Average V1 response elicited by image regions that demarcated object boundaries (red) or were part of the background (blue). Lower panel, response difference. BoM in this condition had a latency

\section{Discussion}

378 We investigated how objects in natural images influence neuronal activity in early visual cortex and observed widespread influences of objecthood on neuronal activity in the human early visual cortex.

These results were mirrored by the early and late modulation of neuronal activity in areas V1 and 
381 V4 of monkeys. Early influences were related to the tuning of the neurons, causing object 382 boundaries to elicit more activity than background elements. However, if we held the image 383 elements in the RF constant, image elements that were part of an object also elicited more activity 384 than elements that were part of the background. This contextual influence manifested during a later 385 phase of the neuronal response, which suggests the involvement of feedback from higher areas 386 and/or horizontal interactions within visual areas. Whereas previous studies on figure-ground 387 segregation and contour integration in early visual cortex used well-controlled, but artificial stimuli, 388 the present results demonstrate that these findings generalize to natural vision. The results are in accordance with theories proposing that image elements of figures are labeled by enhanced neuronal activity in early visual cortex to segregate them from the background ${ }^{10,13,31}$.

Despite the different tasks and recording modalities between humans and monkeys, the neuronal responses in V1 were strikingly similar between the two species (Fig. S2). Our fMRI experiment revealed that BoM for natural images is present in $\mathrm{V} 1$ and other areas of early visual cortex. Object regions evoked stronger response than backgrounds in areas V1 and V2 but OBM did not reach significance in a number of higher areas, including hV4. In contrast, in the electrophysiological experiments in monkeys, OBM was present in V1 but even stronger in V4. This discrepancy may be related to differences between species, experimental setups and differences in spiking versus fMRI measures of neural activity 22,32 . Another relevant difference is the larger size of fMRI pRFs in hV4 compared to neurophysiologically determined V4 RFs. The larger pRF sizes in hV4 may include more neurons with RFs not on the boundary and thereby dilute the BoM signal. Human fMRI allowed us

401 to link the neural responses to human perception, and the monkey neurophysiological experiments 402 allowed us to measure the timing of BoM and OBM and relate it to previous neurophysiological 403 work with synthetic stimuli. 


\section{Early and later object boundary signals}

406 Unexpectedly, natural images elicited BoM during the initial V1 response, at a latency of 50ms. This 407 is much earlier than in previous studies that used well controlled, but artificial stimuli to keep the 408 RF stimulus identical between salient and non-salient contour conditions ${ }^{6,7}$. In these previous studies, the contextual effects on neuronal firing rates were attributed to feedback from higher cortical areas and/or lateral connections within V1, which can inform neurons about information outside the RF. The synaptic and propagation delays associated with these recurrent routes explain why BoM occurs a few tens of ms after the initial V1 response ${ }^{13}$. Our results indicate that the early BoM signals evoked by natural images are not contextual but reflect the tuning of V1 neurons. Indeed, we found that features of object borders differ from those of non-border image regions (Fig. 5c) and that V1 neurons are sensitive to these feature differences (Fig. 5d). On average, the object borders of a particular contrast elicit more activity than non-border image regions with the same contrast. The V1 tuning to object borders is more complex than can be described by Gabor filters $^{25,28,33}$ and is presumably related to a sensitivity to higher-order image statistics ${ }^{34-36}$, which

419 also explain the early detection of boundaries in studies using synthetic figure-ground displays ${ }^{11}$ 420 (Fig. 1b).

421 In addition to their effect on the feedforward response, object boundaries also elicited a contextual 422 influence on V1 activity. When we matched the image elements of object and non-object contours 423 in the RF of V1 neurons, the activity elicited by the object contours was still stronger than that 424 elicited by other, non-object contours (Fig. 6). BoM now occurred at a latency of $81 \mathrm{~ms}$, which is $42530 \mathrm{~ms}$ later than the feedforward response and in line with previous studies that used synthetic 426 stimuli to keep the RF content constant and controlled contour salience by the layout of image 
427 elements in the RF surround ${ }^{6,7}$. This additional delay suggests that BoM now depended on feedback

428 from higher areas and/or horizontal connections within V1. It is of interest that these putative

429 feedback signals increased the activity elicited by contours that are predicted by an object's overall

430 shape. This result is not in accordance with popular "predictive coding" schemes ${ }^{37}$, which suggest

431 that feedback connections should suppress the activity of contours that are predicted by the

432 object's shape. Instead, we found that object borders increase the neuronal activity in the visual

433 cortex, both during the early and later phases of V1 response.

434 BoM is presumably related to border-ownership coding, which is expressed by many neurons in V2,

435 V3, V4 and also by some V1 neurons ${ }^{38-40}$. The activity of neurons with border-ownership signals 436 depends on the side of the figural region relative to the border that falls in the RF. For example, if 437 the border is vertical, some neurons prefer that the border is owned by a figure on the left of it, 438 whereas other neurons have the opposite preference. Hence, border-ownership neurons can link 439 the shape of the border to the surface properties of the object's interior and may therefore play an 440 important role in object recognition. In many situations, the local shape of a border falling in a RF 441 can provide information about the side of the figure ${ }^{24}$. In these situations, neurons express border442 ownership early, during the feedforward response. However, if the RF-stimulus is held constant, 443 border-ownership coding occurs after an additional delay ${ }^{40}$. Although BoM reflects extra activity 444 elicited by the object boundaries compared to less-relevant image elements, and thereby differs 445 from border-ownership coding, it seems likely that the two effects are intimately related. 
448 Image elements that were part of the interior of objects in the scene elicited more activity than 449 background elements, both in human fMRI and monkey neurophysiology. This finding generalizes 450 previous results on the neuronal mechanism of figure-ground perception to natural images (Fig. $4511 b, c)^{10-12,16,41-46}$. Studies using synthetic stimuli revealed a number of successive processing phases 452 for the processing of texture defined figure-ground stimuli (Fig. 1b, reviewed in ref. ${ }^{31}$ ). In V1, the 453 first phase is the arrival of the input from the LGN at a latency of $\sim 40 \mathrm{~ms}$. This is followed at a latency 454 of $\sim 60 \mathrm{~ms}$ by boundary enhancement. Boundaries between figure and ground now elicit extra activity in V1, an effect that starts in the superficial layers of cortex ${ }^{41}$. The change in feature values at a boundary between figure and ground can be detected locally (e.g. there is an abrupt change in 457 the texture as in Fig. 1b) and the mechanisms presumably overlap with early BoM (Fig. 7a). In a yet later phase, at a latency of $\sim 90 \mathrm{~ms}, \mathrm{~V} 1$ neurons that represent the figure's interior enhance their 459 activity. Enhancement in the figure's interior is a genuine contextual effect, because the properties of the image elements that fall into the RF are often not informative about whether they belong to 461 figure and ground (Fig. 1b,c). In these cases, the information that a RF falls on a figure comes from 462 outside the RF. The relatively long latency of this figure-ground modulation is compatible with 463 recurrent loops that may include horizontal connections within V1 and loops through the higher 464 visual areas. Indeed, if activity in higher areas is blocked, figure-ground modulation in the center of 465 the figure is diminished ${ }^{47,48}$, implying an import contribution of recurrent routes through higher 466 visual cortical areas $^{49}$. Interestingly, the optogenetic blockade of the late V1 activity phase with 467 figure-ground modulation selectively impairs figure-ground perception, whereas contrast detection 468 is unimpaired ${ }^{48}$.

469 The activity of image elements that were part of the interior of objects of natural images was 470 enhanced in V1 at a latency of $78 \mathrm{~ms}$, which is $28 \mathrm{~ms}$ after the visually driven response during the 
471 first fixation. In V4 this OBM signal occurred at a latency of 93ms. We also observed systematic

472 differences between the features of object interiors and those in the background, indicating that 473 the tuning of V1 neurons could, in principle, discriminate between features of figure and 474 background, even though the early V1 population response did not exhibit OBM.

Trans-saccadic integration

477 Previous studies demonstrated that figure-ground signals for synthetic stimuli can persist across eye movements ${ }^{17,18}$. As a result, the figure-ground structure that is perceived during one fixation can be quickly reassigned to the appropriate neurons after all RFs shifted across the image due to the saccade. In the present study, OBM occurred sooner after the visually driven response for later fixations than for the first fixation. This result suggests that information about the location of object interiors is indeed carried over to the new fixation ${ }^{18}$, providing insight into the neuronal mechanisms for trans-saccadic integration in natural images ${ }^{17,50,51}$. One possible mechanism for the remapping of these response modulations in early visual cortex are neurons in parietal and frontal cortex that remap salient image elements and could provide feedback to lower areas after each saccade ${ }^{52-54}$. Another possible mechanism is provided by neurons that code the position of objects in nonretinotopic, e.g. head-centered coordinates ${ }^{55,56}$. These cells do not need to update their activity after an eye movement because object position relative to the head is independent of eye position.

489 These neurons could feed the location of objects back to early visual cortex after a coordinate 490 transformation from head to eye centered coordinates, based on the new, post-saccadic eye 491 position. A final source for the early post-saccade OBM are neurons in areas of the temporal stream 492 that code for the overall shape of objects. Many of these neurons are translation invariant, i.e. their 493 activity depends little on the precise location of an object on the retina ${ }^{57}$. These neurons represent 
object shape ${ }^{58}$ and could provide feedback to boost the activity of neurons in lower areas that represent relevant shape features after a saccade.

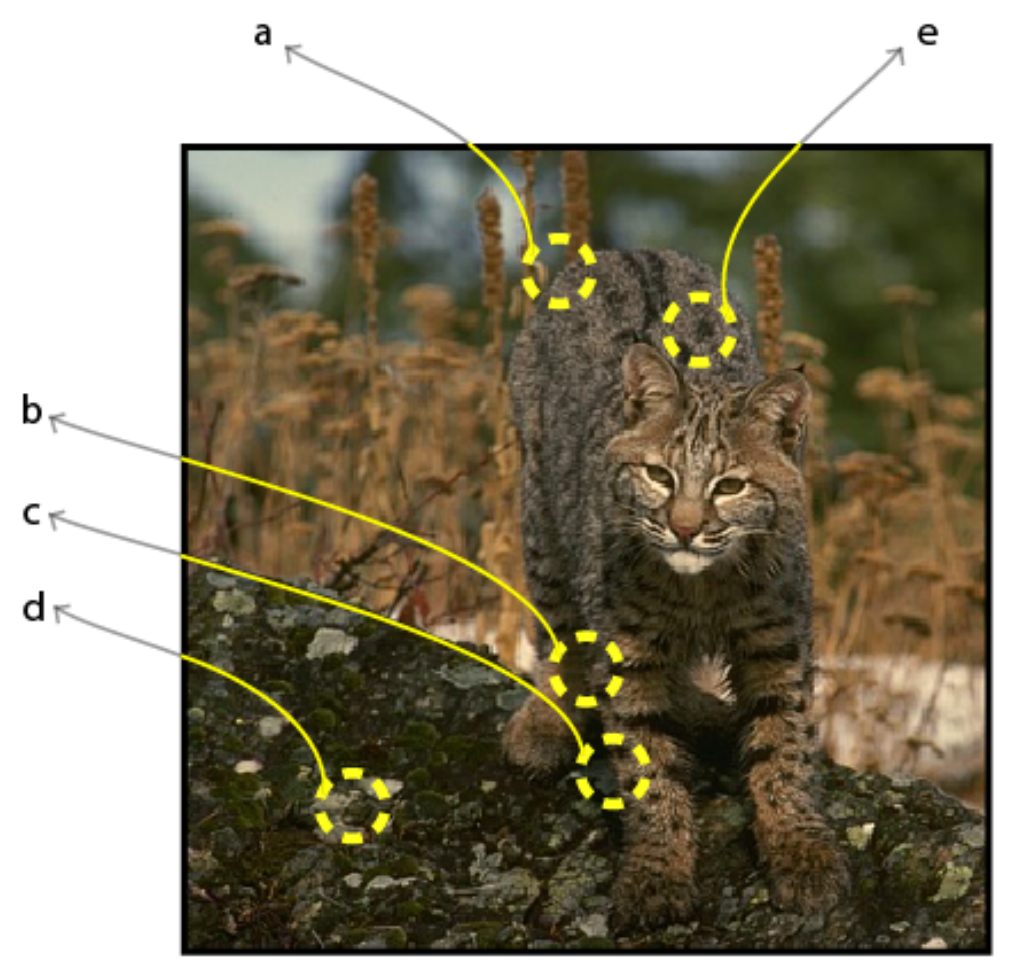

Figure 7. Bottom-up and top-down mechanisms for object detection in natural vision

Summary of the results. The local features of contour a suggest that is an object boundary. It can be detected bottom-up by tuning of V1 neurons. Image patches $\mathbf{b}$ and $\mathbf{c}$ have similar features but the context indicates that $\mathbf{c}$ contains an object boundary and $\mathbf{b}$ does not. Image patches $\mathbf{d}$ and $\mathbf{e}$ have similar features, but only $\mathbf{e}$ is part of the interior of the animal.

\section{Conclusion}

505 We conclude that the object boundaries and object interiors of natural images increase neuronal activity in the visual cortex. The extra neuronal activity occurs early if the local image elements in the RF have a high degree of "objecthood" and at a later point in time if it depends on contextual information outside the RF. OBM and BoM play an important role in perceptual organization ${ }^{13}$, the 
objects and the background by labeling the object features with enhanced neuronal activity ${ }^{13,59-63}$.

511 The presence of these cortical image parsing signals for natural images suggest that they play a role 512 during each fixation of our everyday vision, opening many avenues for future research.

\section{Acknowledgments}

515 We thank Kor Brandsma and Anneke Ditewig for biotechnical support. The work was supported by 516 the European Union's Horizon 2020 and FP7 Research and Innovation Program (Framework 517 Partnership Agreement No. 650003 [HBP FPA]) to PR, the European Union's Erasmus+ program 518 (grant agreement: 2018-1-IT02-KA103-047276/10) to PP and the Netherlands Organization for 519 Scientific Research (NWO) Vidi (452.08.008) and Vici (016.vici.185.050) to SD.

\section{References}

522 1. Martin, D., Fowlkes, C., Tal, D. \& Malik, J. A database of human segmented natural images and its application to evaluating segmentation algorithms and measuring ecological statistics. in Proceedings of the IEEE International Conference on Computer Vision vol. 2 416-423 (Iccv Vancouver:, 2001).

2. Zuiderbaan, W., van Leeuwen, J. \& Dumoulin, S. O. Change Blindness Is Influenced by Both Contrast Energy and Subjective Importance within Local Regions of the Image. Front Psychol 8, 1718 (2017).

3. Neri, P. Object segmentation controls image reconstruction from natural scenes. PLoS Biol 15, e1002611 (2017).

4. Self, M. W., Mookhoek, A., Tjalma, N. \& Roelfsema, P. R. Contextual effects on perceived contrast: figure-ground assignment and orientation contrast. Journal of Vision 15, 2 (2015). 
533 5. Papale, P. et al. Foreground-background segmentation revealed during natural image $534 \quad$ viewing. eNeuro 5, (2018).

535 6. Li, W., Piech, V. \& Gilbert, C. D. Contour saliency in primary visual cortex. Neuron 50, 951$536962(2006)$.

537 7. Chen, M. et al. Incremental integration of global contours through interplay between visual $538 \quad$ cortical areas. Neuron 82, 682-694 (2014).

539 8. Sigman, M., Cecchi, G. A., Gilbert, C. D. \& Magnasco, M. O. On a common circle: natural 540 scenes and Gestalt rules. Proc Natl Acad Sci U S A 98, 1935-1940 (2001).

9. Geisler, W. S., Perry, J. S., Super, B. J. \& Gallogly, D. P. Edge co-occurrence in natural images predicts contour grouping performance. Vision Res 41, 711-724 (2001).

10. Lamme, V. A. The neurophysiology of figure-ground segregation in primary visual cortex. J Neurosci 15, 1605-1615 (1995).

11. Poort, J. et al. The role of attention in figure-ground segregation in areas V1 and V4 of the visual cortex. Neuron 75, 143-156 (2012). Cortex. Cereb Cortex 26, 3964-3976 (2016).

13. Roelfsema, P. R. Cortical algorithms for perceptual grouping. Annu Rev Neurosci 29, 203227 (2006).

14. Roelfsema, P. R. \& Houtkamp, R. Incremental grouping of image elements in vision. Atten Percept Psychophys 73, 2542-2572 (2011).

554 15. Felsen, G. \& Dan, Y. A natural approach to studying vision. Nature Neuroscience 8, 1643555 1646 (2005). 
556 16. Self, M. W. et al. The Segmentation of Proto-Objects in the Monkey Primary Visual Cortex. Curr Biol 29, 1019-1029 e4 (2019).

17. Khayat, P. S., Spekreijse, H. \& Roelfsema, P. R. Correlates of transsaccadic integration in the primary visual cortex of the monkey. Proc Natl Acad Sci U S A 101, 12712-12717 (2004).

18. O'Herron, P. \& von der Heydt, R. Remapping of border ownership in the visual cortex. J Neurosci 33, 1964-1974 (2013).

19. Dumoulin, S. O. \& Wandell, B. A. Population receptive field estimates in human visual cortex. Neurolmage 39, 647-660 (2008).

20. Zuiderbaan, W., Harvey, B. M. \& Dumoulin, S. O. Image identification from brain activity using the population receptive field model. PLOS ONE 12, e0183295 (2017).

21. Arbelaez, P., Maire, M., Fowlkes, C. \& Malik, J. Contour detection and hierarchical image segmentation. IEEE Trans Pattern Anal Mach Intell 33, 898-916 (2011).

22. Self, M. W., van Kerkoerle, T., Goebel, R. \& Roelfsema, P. R. Benchmarking laminar fMRI: Neuronal spiking and synaptic activity during top-down and bottom-up processing in the different layers of cortex. Neurolmage 197, 806-817 (2019).

23. Roelfsema, P. R., Khayat, P. S. \& Spekreijse, H. Subtask sequencing in the primary visual cortex. Proc Natl Acad Sci U S A 100, 5467-5472 (2003).

24. Fowlkes, C. C., Martin, D. R. \& Malik, J. Local figure-ground cues are valid for natural images. Journal of Vision 7, 2 (2007).

25. Walker, E. Y. et al. Inception loops discover what excites neurons most using deep predictive models. Nature Neuroscience (2019) doi:10.1038/s41593-019-0517-x.

26. Bashivan, P., Kar, K. \& DiCarlo, J. J. Neural population control via deep image synthesis. Science (2019) doi:10.1126/science.aav9436. 
579 27. Klindt, D. A., Ecker, A. S., Euler, T. \& Bethge, M. Neural system identification for large populations separating "what" and "where." in Advances in Neural Information Processing Systems (2017). doi:10.12751/nncn.bc2017.0132.

28. Cadena, S. A. et al. Deep convolutional models improve predictions of macaque V1 responses to natural images. PLOS Comput Biol 15, e1006897 (2019).

29. Zhang, Y., Lee, T. S., Li, M., Liu, F. \& Tang, S. Convolutional neural network models of V1 responses to complex patterns. J Comput Neurosci 46, 33-54 (2019).

30. Portilla, J. \& Simoncelli, E. P. A parametric texture model based on joint statistics of complex wavelet coefficients. International Journal of Computer Vision 40, 49-70 (2000).

31. Roelfsema, P. R. \& de Lange, F. P. Early Visual Cortex as a Multiscale Cognitive Blackboard. Annu Rev Vis Sci 2, 131-151 (2016).

32. O'Herron, P. et al. Neural correlates of single-vessel haemodynamic responses in vivo. Nature 534, 378-382 (2016).

33. Tang, S. et al. Complex Pattern Selectivity in Macaque Primary Visual Cortex Revealed by Large-Scale Two-Photon Imaging. Curr Biol 28, 38-48 e3 (2018).

34. Johnson, A. P. \& Baker, C. L. First- and second-order information in natural images: a filterbased approach to image statistics. Journal of the Optical Society of America A 21, 913

35. Dimattina, C. \& Baker, C. L. Modeling second-order boundary perception: A machine learning approach. PLoS Computational Biology 15, e1006829 (2019).

36. Mareschal, I. \& Baker, C. L. A cortical locus for the processing of contrast-defined contours. 
601 37. Friston, K. A theory of cortical responses. Philosophical Transactions of the Royal Society B: Biological Sciences 360, 815-836 (2005).

38. Williford, J. R. \& von der Heydt, R. Figure-Ground Organization in Visual Cortex for Natural Scenes. eNeuro 3, (2016).

605

606

607

608 cortex. J Neurosci 20, 6594-6611 (2000).

40. Hesse, J. K. \& Tsao, D. Y. Consistency of Border-Ownership Cells across Artificial Stimuli, Natural Stimuli, and Stimuli with Ambiguous Contours. J Neurosci 36, 11338-11349 (2016).

41. Self, M. W., van Kerkoerle, T., Super, H. \& Roelfsema, P. R. Distinct roles of the cortical layers of area V1 in figure-ground segregation. Curr Biol 23, 2121-2129 (2013).

42. Scholte, H. S., Jolij, J., Fahrenfort, J. J. \& Lamme, V. A. Feedforward and recurrent processing in scene segmentation: electroencephalography and functional magnetic resonance imaging. J Cogn Neurosci 20, 2097-2109 (2008).

43. Super, H., Spekreijse, H. \& Lamme, V. A. Two distinct modes of sensory processing observed in monkey primary visual cortex (V1). Nature Neuroscience 4, 304-310 (2001).

44. Zipser, K., Lamme, V. A. \& Schiller, P. H. Contextual modulation in primary visual cortex. J Neurosci 16, 7376-7389 (1996).

45. Likova, L. T. \& Tyler, C. W. Occipital network for figure/ground organization. Exp Brain Res 189, 257-267 (2008).

46. Poltoratski, S. \& Tong, F. Resolving the spatial profile of figure enhancement in human V1 through population receptive field modeling. Journal of Neuroscience (2020) doi:10.1523/JNEUROSCI.2377-19.2020. 
47. Klink, P. C., Dagnino, B., Gariel-Mathis, M. A. \& Roelfsema, P. R. Distinct Feedforward and Feedback Effects of Microstimulation in Visual Cortex Reveal Neural Mechanisms of Texture Segregation. Neuron 95, 209-220 e3 (2017).

48. Kirchberger, L. et al. The essential role of recurrent processing for figure-ground perception in mice. Science Advances 7, (2021).

49. Kar, K., Kubilius, J., Schmidt, K., Issa, E. B. \& DiCarlo, J. J. Evidence that recurrent circuits are critical to the ventral stream's execution of core object recognition behavior. Nature Neuroscience 22, 974-983 (2019).

50. McConkie, G. W. \& Currie, C. B. Visual stability across saccades while viewing complex pictures. J Exp Psychol Hum Percept Perform 22, 563-581 (1996).

51. Irwin D.J., D. E. ; G. Eye movements, attention and trans-saccadic memory. J Visual Cognition 5, 127-155 (1998).

52. Gottlieb, J. P., Kusunoki, M. \& Goldberg, M. E. The representation of visual salience in monkey parietal cortex. Nature 391, 481-484 (1998).

53. Duhamel, J. R., Colby, C. L. \& Goldberg, M. E. The updating of the representation of visual space in parietal cortex by intended eye movements. Science 255, 90-92 (1992).

54. Umeno, M. M. \& Goldberg, M. E. Spatial processing in the monkey frontal eye field. I. Predictive visual responses. J Neurophysiol 78, 1373-1383 (1997).

55. Duhamel, J. R., Bremmer, F., ben Hamed, S. \& Graf, W. Spatial invariance of visual receptive fields in parietal cortex neurons. Nature 389, 845-848 (1997).

56. Graziano, M. S., Yap, G. S. \& Gross, C. G. Coding of visual space by premotor neurons. Science 266, 1054-1057 (1994). 
645 57. Tovee, M. J., Rolls, E. T. \& Azzopardi, P. Translation invariance in the responses to faces of 646 single neurons in the temporal visual cortical areas of the alert macaque. J Neurophysiol 72, 1049-1060 (1994).

648 58. Bao, P., She, L., McGill, M. \& Tsao, D. Y. A map of object space in primate inferotemporal cortex. Nature 583, 103-108 (2020).

650 59. Lamme, V. A. \& Roelfsema, P. R. The distinct modes of vision offered by feedforward and recurrent processing. Trends Neurosci 23, 571-579 (2000).

652 60. Houtkamp, R. \& Roelfsema, P. R. Parallel and serial grouping of image elements in visual perception. J Exp Psychol Hum Percept Perform 36, 1443-1459 (2010).

654 61. Pooresmaeili, A. \& Roelfsema, P. R. A growth-cone model for the spread of object-based attention during contour grouping. Curr Biol 24, 2869-2877 (2014).

656 62. Korjoukov, l. et al. The time course of perceptual grouping in natural scenes. Psychol Sci 23, 1482-1489 (2012).

658 63. Jeurissen, D., Self, M. W. \& Roelfsema, P. R. Serial grouping of 2D-image regions with 


\section{Methods}

\section{fMRI experiment with human participants}

\section{Subjects}

Four participants (all male; ages $29-41$ years) participated in the fMRI experiment. All participants had normal or corrected-to-normal visual acuity. We obtained informed written consent of the participants and the protocol was approved by the Human Ethics Committee of University Medical Center Utrecht.

\section{Stimulus presentation}

The visual stimuli were generated in Matlab (Mathworks Inc.) using the PsychToolbox ${ }^{64,65}$ on a Macintosh MacBook Pro. The stimuli were back-projected on a display inside the MRI bore. The subject viewed the display through mirrors inside the scanner. The size of the display was $15.0 \times 7.9 \mathrm{~cm}$ with a resolution of $1024 \times 538$ pixels. The total distance from the subject's eyes to the display was $41 \mathrm{~cm}$. The stimuli were constrained to a circular area (radius, $5.5^{\circ}$ ) with the size of the vertical dimension of the screen. The area outside this circle was maintained at a constant mean luminance.

\section{Population receptive field (pRF) mapping stimulus}

We used bar apertures filled with natural images ${ }^{19,20}$ (Fig. S2) to train the pRF-model. The width of the bar subtended $1 / 4$ th of the stimulus radius $\left(1.375^{\circ}\right)$. Four bar orientations $\left(0^{\circ}, 45^{\circ}, 90^{\circ}\right.$ and $\left.135^{\circ}\right)$ and two different step directions for each bar were used, giving a total of 8 bar directions within a given scan. The bar stepped across the stimulus aperture in 20 steps (with a distance of $0.55^{\circ}$ and a 
duration of 1.5 seconds per bar position) so that each pass took 30 seconds. A period of 30 seconds mean-luminance ( $0 \%$ contrast) was presented after every pass. In total there were 4 blocks of mean-

687 luminance during each scan, presented at evenly spaced intervals. The participants performed a 688 fixation dot task to make sure they fixated at the center of the display. A small fixation dot $\left(0.11^{\circ}\right.$ 689 radius) was presented in the middle of the stimulus. The fixation dot changed its color from red to green at random time intervals and subjects were instructed to respond to color changes using a button press.

\section{Natural images}

694 The natural images came from the BSD ${ }^{1,21}$. The original resolution of the images was $321 \times 481$ pixels 695 (both landscape and portrait). In the fMRI experiments ${ }^{20}$, we selected a square region of $321 \times 321$ 696 pixels from the images and upsampled it to a resolution of 516x516 pixels, which corresponds to a 697 stimulus of $11 \times 11^{\circ}$ diameter of visual angle. The images were masked by a circle with a raised cosine 698 faded edge (width of $0.9^{\circ}$ ), and the areas outside this circle were set to the mean luminance. The 699 images were gamma-linearized and the mean contrast was set to 50\%. We used 3 image sets in 700 different scanning runs, each containing 15 different natural images (45 in total) and one full-field 701 binarized bandpass-filtered noise stimulus. Figure S1 shows the image set. A fixation dot was 702 presented at the center of the stimulus. We used the same fixation dot task as for the pRF mapping 703 runs.

\section{Functional imaging and processing}

706 The MRI data was acquired with a Philips 7T scanner using a 32-channel head-coil ${ }^{20}$. We scanned 707 the participants with a 2 d-echo-planar-imaging sequence with 25 slices oriented perpendicular to 
708 the calcarine sulcus with no gap. The following parameters were used; repetition time $(T R)=$ $7091500 \mathrm{~ms}$, echo time (TE) $=25 \mathrm{~ms}$ and a flip angle of $80^{\circ}$. The functional resolution was $2 \times 2 \times 2 \mathrm{~mm}$ and 710 the field of view (FOV) was $190 \times 190 \times 50 \mathrm{~mm}$. We used foam padding to minimize head movement.

711 The functional images were corrected for head movement between and within the scans ${ }^{66}$. For 712 computation of the head movement between scans, the first functional volumes for each scan were 713 aligned. Within scan motion correction was then computed by aligning the frames of a scan to the 714 first frame. The duration of the pRF mapping scans was 372 seconds (248 time-frames), of which the first 12 seconds (8 time-frames) were discarded to eliminate start-up magnetization transients. During the three sessions we acquired 6-8 pRF mapping scans in total per subject. To obtain a high signal-to-noise ratio, we averaged across the repeated scans. During the three sessions in which we presented the natural images we acquired 6-7 scans for each of the three stimulus sets. The duration of the scans with the natural images was 432 seconds (288 time-frames). The first 12 seconds ( 8 time-frames) were discarded to eliminate start-up magnetization transients. The images were presented in a block design. Each image was presented during a 9-second block. Within this block the same image was shown 18 times for a duration of $300 \mathrm{~ms}$ followed by $200 \mathrm{~ms}$ mean-luminance.

723 The full-field stimuli were presented with 3 alternating different high-contrast patterns, to obtain a 724 full high-contrast response that is not based upon one specific high-contrast pattern (Fig. S2b). 725 Specifically, the phase of the full-field pattern was randomized on different presentations in order to obtain a response that is not influenced by one specific dartboard pattern. The block in which the stimulus was presented was followed by a 12 second mean-luminance presentation. Four longer 728 blank periods of 33 seconds were also included during the scan. 
731 The T1-weighted MRI images were acquired in a separate session using an 8-channel SENSE head732 coil. The following parameters were used: TR/TE/flip angle $=9.88 / 4.59 / 8$. The scans were acquired 733 at a resolution of $0.79 \times 0.80 \times 0.80 \mathrm{~mm}$ and were resampled to a resolution of $1 \mathrm{~mm}^{3}$ isotropic. The 734 functional MRI scans were aligned with the anatomical MRI using an automatic alignment 735 technique ${ }^{66}$. From the anatomical MRI, white matter was automatically segmented using the 736 FMRIB's Software Library $(F S L)^{67}$. After the automatic segmentation it was hand-edited to minimize segmentation errors ${ }^{68}$. The gray matter was grown from the white matter to form a $4 \mathrm{~mm}$ layer surrounding the white matter. A smoothed 3D cortical surface can be rendered by reconstruction of the cortical surface at the border of the white and gray matter ${ }^{69}$.

742 The pRF-model was estimated for every cortical location from the measured fMRI signal that was 743 elicited by the pRF mapping bar stimuli (Fig. S2a) ${ }^{19,20}$. In short, the method estimates the pRF by 744 combining the measured $\mathrm{fMRI}$ time-series with the position time course of the visual stimulus. A 745 prediction of the time-series is made by calculating the overlap of the pRF and the stimulus energy 746 (RMS contrast, see below) convolved with the hemodynamic response function (HRF). We 747 estimated the parameters of the HRF that best describes the data of the whole acquired fMRI 748 volume ${ }^{70}$. The optimal parameters of the pRF-model are chosen by minimizing the residual sum of 749 squares between the predicted and the measured time-series. We used the conventional pRFmodel, which consists of a circular symmetric Gaussian. This model has four parameters: position $751(x, y)$, size $(\sigma)$ and amplitude $(\beta)$. For further technical and implementation details see ${ }^{19}$. 
754 We used the pRF-method to estimate position parameters $\mathrm{x}$, and $\mathrm{y}$ of the pRF of every voxel. From 755 these values, we derived the polar angle $\left(\operatorname{atan}\left(\mathrm{y}_{0} / \mathrm{x}_{0}\right)\right)$ and eccentricity $\left(\mathrm{V}\left(\mathrm{x}_{0}{ }^{2}+\mathrm{y}_{0}{ }^{2}\right)\right)$ values. We drew 756 the borders between visual field maps on the basis ${ }^{71}$ polar angle and eccentricity maps on the 757 inflated cortical surface ${ }^{69}$. We defined visual areas V1, V2, V3, hV4, LO-1/2 and V3-a/b as our regions 758 of interest (ROIs) ${ }^{71-74}$.

Analysis of fMRI responses to the natural images

761 We measured fMRI responses to 45 natural images (Fig. S1) and 3 full-field high contrast stimuli $(100 \% \text { contrast; Figure S2 })^{20}$. We first determined the voxel response amplitudes in \%BOLD signal change elicited by each of these images. The voxel responses were calculated using a general linear model $(G L M)^{75,76}$. To reduce the noise from the individual voxel differences in response amplitudes, we normalized the responses to the voxel's response to the full-field ( $100 \%$ contrast) stimulus. significant response ( $t$-values $>4.0$ ), a pRF eccentricity between 0.5 and $4^{\circ}$ and for which the pRF model explained more than $40 \%$ of the variance. Based on previous work, for every area we used a threshold for the pRF sizes ${ }^{19,70,77,78}$. In V1 we included pRFs with a value of $\sigma$ (which determines pRF size) between $0.25^{\circ}$ and $0.8^{\circ}$, for $\mathrm{V} 2$ between $0.25^{\circ}$ and $1.1^{\circ}$, for $\mathrm{V} 3$ between $0.25^{\circ}$ and $1.75^{\circ}$, for hV4 between $0.45^{\circ}$ and $3^{\circ}$, for $\mathrm{V} 3 a b$ between $0.45^{\circ}$ and $3.75^{\circ}$ and for and LO12 between $0.9^{\circ}$ and $5^{\circ}$.

773 To derive the CRF, we computed the contrast of every natural image within each pRF. The pRF of 774 voxels was modeled as a circular symmetric Gaussian function, described by parameters for position $775\left(x_{c}, y_{c}\right)$ and size $(\sigma)$, giving rise to a Gaussian weighting function $w_{i}$ : 


$$
w_{i}=\exp \left(\frac{\left(x_{i}-x_{c}\right)^{2}+\left(y_{i}-y_{c}\right)^{2}}{2 \sigma^{2}}\right)
$$
parameters).

Quantification of BOM and OBM

The BSD images are annotated by 5-9 human observers who drew lines to identify borders that are

Where $x_{c}$ and $y_{c}$ define the location of the center of the pRF in the visual field, $\sigma$ determines the size of the pRF and $x_{i}$ and $y_{i}$ define the location of the $i$-th pixel. We computed each voxel's contrast value to each natural image by calculating the Root-Mean-Squared (RMS) contrast ${ }^{65,79}$ of the part of the image inside the voxel's pRF. RMS contrast was defined as the standard deviation of the luminance of the pixels relative to the mean. The RMS-contrast was weighted by the pRF Gaussian function to obtain the local contrast-energy value per pRF:

Where $N$ is the number of pixels in the stimulus window. $L$ is the mean luminance from the pixels inside the spatial window, and $L_{i}$ is the luminance of the $i$-th pixel ${ }^{2}$.

We computed the CRF of voxels areas V1, V2, V3, hV4, LO-1/2 and V3-a/b by measuring the fMRI responses as a function of the contrast inside the pRF. We chose contrast bins such that every bin contained $10 \%$ of the voxels and fitted the following equation (modified from ref. ${ }^{80}$ ):

$$
R(C)=a \frac{C^{q}}{C^{q}+Q^{q}}
$$

where $\mathrm{R}$ is the $\mathrm{fMRI}$ response, $C$ is the RMS-contrast inside the $p R F, Q$ represents the contrast value where the CRF is at half of its maximum response, and $q$ determines the slope $(Q$ and $q$ are free

important for the scene's representation ${ }^{1,21}$. We used these measurements to define the perceived 
797 boundaries, which are salient boundaries of the scene. Every pixel $i$ of the manually labeled images 798 have values for the degree of agreement between observers, $S_{i}$, between 0 (not labeled by any 799 observer) and 1 (labeled by all observers). The border-salience in the pRF is calculated as a weighted 800 sum across pixels:

$$
\text { Perceived border }=\frac{\sum_{i=1}^{N} w_{i} \cdot S_{i}}{\sum_{i=1}^{N} w_{i}}
$$

Here $w_{i}$ are the weights of the RF estimate (equation 1 ) and $N$ is the total number of pixels in the

RF. We used the same method to quantify the degree to which a pixel was part of an object or the background (Fig. 1e). Pixels that were as part of an object, had a value of 1 and pixels that were part of the background had a value of 0 . We selected a segmentation covering the objects of a scene from one of the BSD subjects, and then considered everything else as background 5 . We excluded 3 of the 45 images in the OBM analysis because the object in the image almost filled the entire scene.

We split the voxels based on objecthood values inside the pRFs. We included the lowest 25 percent responses as non-perceived borders/background and the highest 25 percent as perceivedborders/object-interior and computed the CRFs within these voxel classes.

\section{Statistics}

We used a bootstrapping procedure to determine the significance of differences in CRFs between conditions. We sampled the images with replacement 1000 times, fit the CRF for the two simulated conditions and computed the mean difference. We derived the $p$-value from this null distribution. 
819 All procedures complied with the NIH Guide for Care and Use of Laboratory Animals and were 820 approved by the institutional animal care and use committee of the Royal Netherlands Academy of 821 Arts and Sciences. Two macaque monkeys (males, 7 and 13 years old) participated in the 822 electrophysiological experiments. They were socially housed in stable pairs in a specialized primate 823 facility with natural daylight, controlled humidity and temperature. The home-cage was a large 824 floor-to-ceiling cage which allowed natural climbing and swinging behavior. The cage had a solid 825 floor, covered with sawdust and was enriched with toys and foraging items. Their diet consisted of monkey chow supplemented with fresh fruit. Their access to fluid was controlled, according to a carefully designed regime for fluid uptake. During weekdays the animals received water or diluted 828 fruit juice in the experimental set-up upon correctly performed trials. We ensured that the animals drank sufficient fluid in the set-up and supplemented extra fluid after the recording session if they did not drink enough. On days of the weekend, they received at least $700 \mathrm{ml}$ water in the home-cage in a drinking bottle. The animals were regularly checked by veterinary staff and animal caretakers and their weight and general appearance were recorded daily in an electronic logbook during fluidcontrol periods.

\section{Surgical details}

We implanted both monkeys with a titanium head-post (Crist instruments) under aseptic conditions and general anesthesia as reported previously ${ }^{81}$. The monkeys were trained to direct their gaze to a underwent a second operation to implant 5x5 arrays of micro-electrodes (Utah-probes, Blackrock Microsystems) over opercular V1 and V4. The inter-electrode spacing of the arrays was $400 \mu \mathrm{m}$. We obtained good signals from $4 \mathrm{~V} 1$ arrays in each monkey and from $2 \mathrm{~V} 4$ arrays in monkey $\mathrm{B}^{11}$. 


\section{Electrophysiology}

We recorded neuronal activity of 192 recording sites in V1 (96 in Monkey M and 96 in Monkey B) and $48 \mathrm{~V} 4$ recording sites in monkey $\mathrm{B}$. We recorded the envelope of multi-unit activity by digitizing the signal referenced to a subdural electrode at $24.4 \mathrm{kHz}$. The signal was band-pass filtered (2nd

rectified (negative becomes positive) and low-pass filtered (corner frequency $=200 \mathrm{~Hz}$ ) to produce the envelope of the high-frequency activity, which we refer to as MUA ${ }^{82}$. The MUA signal reflects the population spiking of neurons within $100-150 \mu \mathrm{m}$ of the electrode and the population responses are very similar to those obtained by pooling across single units ${ }^{82-85}$.

\section{Receptive Field Mapping}

854 We mapped the RF of each recording site in V1 using a drifting luminance-defined bar that moved 855 in one of four directions. The response to each direction was fitted with a Gaussian function. The borders of the RF were then calculated as described previously ${ }^{82}$. The signal-to-noise ratio (SNR $\mathrm{RF}_{\mathrm{RF}}$

857 of the response was taken as the peak of the Gaussian divided by the standard deviation of the pre858 trial baseline response. We only included recording sites in the analyses with a reliable visual 859 response (i.e., the responses to all four bar directions had an SNRRF of at least 1). The median V1 RF 860 size, taken as the square-root of the area, was $1.8^{\circ}$ (range $0.4^{\circ}$ to $8.2^{\circ}$ ) and the median eccentricity 861 of the RFs was $2.4^{\circ}$ (range $0.6^{\circ}$ to $12.9^{\circ}$ ). We mapped V4 RFs by presenting white dots $\left(0.5^{\circ}\right.$, 862 luminance $82 \mathrm{~cd} \cdot \mathrm{m}^{-2}$ ) on a gray background (luminance $14 \mathrm{~cd} \cdot \mathrm{m}^{-2}$ ) at different positions of a grid $863\left(0.5^{\circ}\right.$ spacing $)$. The hotspot of the V4 RF was defined as the position with the maximum response 864 (median eccentricity $4.04^{\circ}$, range $0.79^{\circ}-7.43^{\circ}$ ) and the RF borders as the locations where activity 
865 fell below $50 \%$ of the maximum ${ }^{86}$. Using this criterion, the median V4 RF size was $4.5^{\circ}$ (range $2.6^{\circ}-$ $\left.866 \quad 6.0^{\circ}\right)$

\section{Stimulus presentation}

869 In the experiments with monkeys, stimuli were presented on a CRT monitor at a refresh rate of $60 \mathrm{~Hz}$ 870 and resolution of $1024 \times 768$ pixels viewed from a distance of $46 \mathrm{~cm}$. The monitor had a width of $87140 \mathrm{~cm}$, yielding a field-of-view of $41.6 \times 31.2^{\circ}$. All stimuli were generated in Matlab using the COGENT 872 graphics toolbox (developed by John Romaya at the LON at the Wellcome Department of Imaging 873 Neuroscience). The eye position was recorded using a digital camera (Thomas recordings, $250 \mathrm{~Hz}$ 874 frame-rate).

\section{Selection of recording sites and inclusion of data}

877 To normalize MUA, we first subtracted the mean activity in the pre-trial period in which the animal 878 was fixating (200 to $0 \mathrm{~ms}$ relative to stimulus onset) and divided by the maximum smoothed ( $26 \mathrm{~ms}$ 879 Gaussian kernel) peak response (0-150ms after stimulus onset). In the experiment with multiple 880 saccades, each trial contained multiple fixations and neuronal activity was normalized to the peak 881 response elicited by stimulus onset during the first fixation. The data are therefore in normalized 882 units, where e.g. a value of 0.1 indicates $10 \%$ of the maximal MUA onset response. We only included 883 recording sites on days with a sufficient signal-to-noise ratio (SNR $\left.R_{D A Y}\right)$. SNR DAY $_{\text {was }}$ estimated by 884 dividing the maximum of the initial peak response by the standard deviation of the baseline activity 885 across trials. When the SNR $R_{\text {DAY }}$ of a recording site was smaller than 2 on particular day, we removed 
conditions and to compute the CRFs, MUA activity was generally averaged in a $0-300 \mathrm{~ms}$ time window. Analyses with different time-windows have been specified in the main text.

891 To compute the latency of neural responses a function was fitted to the time-course of interest (i.e.

892 the difference between object borders and non-border image regions or the difference between 893 the object interior and background $)^{11,12,23}$. The function was derived from the assumptions that the onset of the response has a Gaussian distribution and that a fraction of the response dissipates exponentially which yields the following equation:

$$
f(t)=d \cdot \exp \left(\mu \alpha+0.5 \sigma^{2} \alpha^{2}-\alpha t\right) \cdot\left(G\left(\mathrm{t}, \mu+\sigma^{2} \alpha, \sigma\right)+c \cdot G(\mathrm{t}, \mu, \sigma)\right.
$$

Where $G(t, \mu, \sigma)$ is a cumulative Gaussian density with mean $\mu$ and standard deviation $\sigma, \alpha^{-1}$ is the time constant of the dissipation, and $c$ and $d$ represent the contribution the non-dissipating and dissipating components, respectively. The latency was defined as the point at which the fitted function reached $33 \%$ of its maximum. To compare the latency of the BoM and OBM between fixation 1 and fixations 2-6, we first subtracted from the OBM latency for each recording site from the latency of visually driven response and performed a Wilcoxon signed rank test (Fig. S7). The latency of visually driven response was computed as the difference between the response elicited by images with the highest and lowest contrast levels in the RF.

Four BSD images from the $\mathrm{fMRI}$ experiment were used in the electrophysiological experiments in 
910 from uniformly spaced grid (with 500 positions) covering the circular aperture of the image. The

911 image appeared once the monkey had maintained fixation for $300 \mathrm{~ms}$ (fixation 1). After an additional $912400 \mathrm{~ms}$, the first fixation point disappeared and another fixation point appeared, at a position 913 sampled from the same grid. The monkey made a saccade to the new fixation point and maintained 914 fixation for an additional 400ms. This fixation-saccade procedure was repeated five times (fixations 915 2-6). Reward was delivered after every correct fixation, with an extra amount at the end of the trial, 916 i.e. after the $6^{\text {th }}$ correct fixation. Aborted trials (i.e., when the monkeys did not maintain fixation for $917400 \mathrm{~ms}$ or did not perform a saccade within $700 \mathrm{~ms}$ ) were repeated at the end. The same image was 918 presented in multiple recording days until data for five repetitions of each grid point for every 919 fixation number was collected. We included data from all correct fixations (e.g., if the trial was 920 interrupted after five fixations, we included the first four). Between the trials, the monkeys 921 occasionally also fixated on parts of the image for longer than $300 \mathrm{~ms}$, and we also included these 922 spontaneous fixations in the analysis. We collected a total of 11,783 correct trials for monkey $M$ and 92313,373 for monkey B, for a total of 50,849 fixations analyzed for monkey $M$ and 60,211 for monkey B.

Data analysis

927 We determined the coordinates of the RF on the image for every fixation and analyzed the data 928 from the first fixation and later fixations separately. We computed contrast, BoM and OBM in the 929 RF, as described above. To quantify the independent influence of object borders, object interiors 930 and contrast, we carried out a variance partitioning analysis ${ }^{87}$. For each recording site, we 931 determined how much variance $\left(R^{2}\right)$ was explained by RMS contrast, object borders and object 932 interiors with independent linear regressions, and by combinations of the three predictors in 
933 where $R_{f u l l}^{2}$ is the variance explained by the full model, including all the predictors, while $R_{B O M+O B M}^{2}$ is the variance explained by the model including BoM and OBM as predictors while leaving contrast out. Similar equations were derived for the FEV accounted for by BoM and OBM.

939 The explained variance estimates were then averaged across recording sites. The full model explained $6.4 \%$ of the variance in V1 for fixation 1 (mean across recording sites), $4.3 \%$ for fixations condition presented in the main text were normalized to these values (see equation 6).

\section{RF models and the prediction of perceived borders} and neuronal tuning (Fig. S9).

We extracted the activity of units of VGG-19's layer conv3_1 (state of the art in predicting V1 responses to natural images ${ }^{28,29}$ ) and followed the approach of ref. ${ }^{28}$ with two modifications. We used a two-step mapping ${ }^{26,27}$, described by following the equation:

$$
r=f_{V G G-19}(\text { input }) * W_{s} * W_{d}
$$

where $r$ is the predicted response of V1 recording site, $f_{V G G-19}($ input) is the output of VGG-19's

953 conv3_1 to our stimulus set (i.e. input), and $W_{s}$ and $W_{d}$ are two sets of weights defining spatial and 
954 feature selectivity, respectively. The spatial mask ( $W_{s}$, initialized as the 2D Gaussian RF estimate) 955 approximates the RF and a weighted sum of the nodes in the ANN $\left(W_{d}\right)$ approximates the feature 956 selectivity of the recorded neurons ${ }^{26}$. We trained the model to optimize $W_{s}$ and $W_{d}$ to predict V1 957 responses to the training set (i.e., input in eq. 7). The activity of V1 recording sites depends on a 958 small and localized portion of the input and we therefore cropped the RF models around the most 959 active pixels (3 SD or more away from the global mean) following the procedure in ref. ${ }^{25}$. To visualize 960 tuning (Fig. 5b,c), we kept $W_{s}$ and $W_{d}$ constant and varied input to maximize the response of the 961 model for a particular V1 recording site. We used cross-validation to assess the quality of the fit as in ref. ${ }^{25}$. Specifically, we used 5,000 trials for training and 100 trials for cross-validation. We trained the model for 300 epochs with a batch size of 256 , using $10 \%$ of the training set for validation.

964 To estimate how well the V1 RF models could detect perceived borders (F-stat metric; Fig. 5b), we 965 first convolved the RF models with unseen images from the BSD test set (100 grayscale images), and matched them with the annotated versions ${ }^{21}$. For simplicity, we defined the border detection 967 performance (BoP) as the F-measure, employed by the authors of the BSD for benchmark 968 evaluation, which we computed using the MATLAB code associated with the dataset: 969 (https://www2.eecs.berkeley.edu/Research/Projects/CS/vision/bsds/code/) ${ }^{20}$. We estimated the 970 chance-level performance in border-detection with a (null) permutation distribution (horizontal 971 dashed line in Fig. 5c; 97.5\% level the distribution), shuffling the class labels (after 72 iterations, one 972 for each recording site included in this analysis. A generalized Pareto distribution was fit to the tail 973 of the permutation distribution ${ }^{88}$. The models were implemented using custom Python code using 974 NumPy ${ }^{89}$, SciPy (SciPy.org), Tensorflow $1.5^{90}$ and with modules from 975 https://github.com/dicarlolab/npc ${ }^{26}$ and https://github.com/sacadena/Cadena2019PlosCB ${ }^{28}$. 
Perceived borders and object interior detection of a population of V1 neurons

978 To examine the strength of BoM and OBM signals across a larger population of V1 recording sites 979 (Fig. 5d), we trained SVMs to discriminate between object borders and non-border image regions 980 based on the activity of 19 recording sites with RFs smaller than $1.5^{\circ}$. We also trained them to 981 distinguish between object interiors and the background. We used 2 of the 4 images for training and 982 the other two for cross-validation.

Neuronal activity profiles across the images

985 To examine the overall activity level elicited by the images (Fig. 3b), we multiplied the activity by

2d-Gaussian approximation of the RFs, weighted by sampling of the visual space caused by the overall pattern of fixations $\mathrm{s}^{5,20,91-95}$ at 7 time points (from 0 to $300 \mathrm{~ms}$ in $50 \mathrm{~ms}$ steps) and activity was averaged within a $25 \mathrm{~ms}$ window centered on each time-point.

\section{Statistics}

991 We compared differences between the CRFs between object borders and non-border image regions 992 and between object interiors and the background using a bootstrapping procedure $(1,000$ 993 iterations), as described for the fMRI data above. To test for differences in the median latencies of 994 BoM and OBM between regions and conditions, we used a signed-rank Wilcoxon test across 995 recording sites. The significance of the Pearson's correlation between BoM at the peak of response 996 and the segmentation performance across V1 recordings sites (Fig. 5b) was assessed with a t-test. 
To test whether isolated image patches from the BSD that were either centered on object borders or not elicited a different level of V1 activity, we carried out an additional experiment in monkey B (50 recording sites, Fig. 5e-g). We chose three V1 recording arrays and centered 100 patches of the image from the BSD that contained object contours and 100 patches that did not contain object contours on the RFs. These patches were automatically selected so that the RMS contrast was the same $(70 \pm 1 \%)$ and the size matched to the median RF of the recording sites of the array $\left(0.9^{\circ}-2.0^{\circ}\right)$. The patches were presented on a grey background $\left(26.8 \mathrm{~cd} \cdot \mathrm{m}^{-2}\right)$ while the monkey maintained gaze on a red fixation point for $300 \mathrm{~ms}$. We repeated each stimulus five times and collected a total of 3,000 trials (1,000 trials per array). We tested the significance of the difference in the activity elicited by isolated object and non-object contour patches at the peak of the response $(25-75 \mathrm{~ms})$ with a Wilcoxon signed rank test across recording sites.

\section{Contextual BoM experiment}

To examine differences in activity elicited by object and non-object contours when the stimulus in the RF was held constant (Figure 6) we selected twelve images from the BSD, which were cropped and upsampled to $512 \times 512$ pixels $\left(23.2^{\circ} \times 23.2^{\circ}\right)$. We ensured that the portion of the image covered by the RF of each recording site and its surround were exactly the same across conditions (same size and content, Fig. 6), so that border salience only depended on information outside the neurons' RF. We used a 2x2 design. The first factor was whether the image element in the RF fell on an object border (Fig. 6a). The second factor was whether we presented the original image or a scrambled version (also known as metamer). To this aim, we created three further stimuli from each image. First, we copied a circular patch (80 pixels in diameter, $3.7^{\circ}$ ) from an object contour location onto a non-object contour location using Adobe Photoshop (blue circle in Fig. 6a, see Fig. S10 for other 
$\llcorner 022$

$\llcorner 023$

$\llcorner 024$

example images). The border of this circular patch was smoothed to blend it in at the new location. We created two metamers using the algorithm of ref. ${ }^{96}$, with Matlab code provided by the authors (https://github.com/freeman-lab/metamers). The two metamers were constructed so that either the object- or non-object contour was kept intact, with a smooth transition to the surround.

Trials started with a red fixation point and the stimulus appeared after $300 \mathrm{~ms}$ of fixation. The monkeys maintained fixation for an additional 400ms after stimulus onset (Fig. 6b). We ensured that the RFs of V1 recording sites were centered on the image patch, which was identical in the four conditions. The order of the conditions was randomized across trials and aborted trials (when the monkeys broke fixation) were repeated at the end. We collected a total of 8,094 trials in monkey $\mathrm{M}$ and 9,111 in monkey $B$.

We tested the significance of the BoM in a window from $0-300 m s$ after stimulus onset (subtracting spontaneous activity, $-100-0 \mathrm{~ms}$ ) with a Wilcoxon signed rank test across recording sites. We also used a repeated-measures two-way ANOVA across recording sites, with object/non-object contour and scrambled/not scrambled as factors.

\section{Data availability}

Data will be available upon publication of the paper.

\section{Code availability}

Custom code will be available upon publication of the paper.

\section{References}

64. Brainard, D. H. The Psychophysics Toolbox. Spat Vis 10, 433-436 (1997). 
L045 65. Pelli, D. G. The VideoToolbox software for visual psychophysics: transforming numbers into movies. Spat Vis 10, 437-442 (1997).

L047 66. Nestares, O. \& Heeger, D. J. Robust multiresolution alignment of MRI brain volumes. Magn Reson Med 43, 705-715 (2000).

67. Smith, S. M. et al. Advances in functional and structural MR image analysis and implementation as FSL. Neurolmage 23 Suppl 1, S208-19 (2004).

68. Yushkevich, P. A. et al. User-guided 3D active contour segmentation of anatomical structures: significantly improved efficiency and reliability. Neurolmage 31, 1116-1128 (2006).

69. Wandell, B. A., Chial, S. \& Backus, B. T. Visualization and measurement of the cortical

70. Harvey, B. M. \& Dumoulin, S. O. The relationship between cortical magnification factor and population receptive field size in human visual cortex: constancies in cortical architecture. J Neurosci 31, 13604-13612 (2011).

71. Wandell, B. A., Dumoulin, S. O. \& Brewer, A. A. Visual field maps in human cortex. Neuron

72. DeYoe, E. A. et al. Mapping striate and extrastriate visual areas in human cerebral cortex. Proc Natl Acad Sci U S A 93, 2382-2386 (1996).

73. Engel, S. A., Glover, G. H. \& Wandell, B. A. Retinotopic organization in human visual cortex and the spatial precision of functional MRI. Cereb Cortex 7, 181-192 (1997).

74. Sereno, M. I. et al. Borders of multiple visual areas in humans revealed by functional magnetic resonance imaging. Science 268, 889-893 (1995). 
L068 76. Friston, K. J. et al. Event-related fMRI: characterizing differential responses. Neurolmage 7, 30-40 (1998).

77. Zuiderbaan, W., Harvey, B. M. \& Dumoulin, S. O. Modeling center-surround configurations in population receptive fields using fMRI. Journal of Vision 12, 10 (2012).

78. Dumoulin, S. O. \& Knapen, T. How Visual Cortical Organization Is Altered by Ophthalmologic and Neurologic Disorders. Annual Review of Vision Science 4, 357-379 (2018).

79. Bex, P. J. \& Makous, W. Spatial frequency, phase, and the contrast of natural images. J Opt Soc Am A Opt Image Sci Vis 19, 1096-1106 (2002).

80. Boynton, G. M., Demb, J. B., Glover, G. H. \& Heeger, D. J. Neuronal basis of contrast discrimination. Vision Res 39, 257-269 (1999).

81. Self, M. W., Kooijmans, R. N., Super, H., Lamme, V. A. \& Roelfsema, P. R. Different glutamate

82. Super, H. \& Roelfsema, P. R. Chronic multiunit recordings in behaving animals: advantages and limitations. Prog Brain Res 147, 263-282 (2005).

83. Cohen, M. R. \& Maunsell, J. H. Attention improves performance primarily by reducing interneuronal correlations. Nature Neuroscience 12, 1594-1600 (2009).

84. Palmer, C., Cheng, S. Y. \& Seidemann, E. Linking neuronal and behavioral performance in a reaction-time visual detection task. J Neurosci 27, 8122-8137 (2007).

85. Trautmann, E. M. et al. Accurate Estimation of Neural Population Dynamics without Spike Sorting. Neuron 103, 292-308 e4 (2019).

86. Motter, B. C. Focal attention produces spatially selective processing in visual cortical areas V1, V2, and V4 in the presence of competing stimuli. J Neurophysiol 70, 909-919 (1993). 
L091 87. Lescroart, M. D., Stansbury, D. E. \& Gallant, J. L. Fourier power, subjective distance, and object categories all provide plausible models of BOLD responses in scene-selective visual areas. Front Comput Neurosci 9, 135 (2015).

88. Knijnenburg, T. A., Wessels, L. F. A., Reinders, M. J. T. \& Shmulevich, I. Fewer permutations, more accurate P-values. Bioinformatics 25, i161-i168 (2009).

89. van der Walt, S., Colbert, S. C. \& Varoquaux, G. The NumPy array: A structure for efficient numerical computation. Computing in Science and Engineering 13, 22-30 (2011).

90. Abadi, M. et al. TensorFlow: Large-Scale Machine Learning on Heterogeneous Distributed Systems. (2016).

91. Thirion, B. et al. Inverse retinotopy: inferring the visual content of images from brain activation patterns. Neurolmage 33, 1104-1116 (2006).

92. Stanley, G. B., Li, F. F. \& Dan, Y. Reconstruction of natural scenes from ensemble responses in the lateral geniculate nucleus. J Neurosci 19, 8036-8042 (1999).

93. Kay, K. N., Naselaris, T., Prenger, R. J. \& Gallant, J. L. Identifying natural images from human brain activity. Nature 452, 352-355 (2008).

94. Miyawaki, Y. et al. Visual image reconstruction from human brain activity using a combination of multiscale local image decoders. Neuron 60, 915-929 (2008).

96. Freeman, J. \& Simoncelli, E. P. Metamers of the ventral stream. Nature Neuroscience 14,

95. Nishimoto, S. et al. Reconstructing visual experiences from brain activity evoked by natural movies. Current Biology 21, 1641-1646 (2011). 